\title{
Glueball-quarkonia content and decay of scalar-isoscalar mesons
}

\author{
M. Strohmeier-Prešiček ${ }^{1}$, T. Gutsche ${ }^{1}$, R. Vinh Mau ${ }^{2}$ and Amand Faessler ${ }^{1}$ \\ ${ }^{1}$ Institut für Theoretische Physik, Universität Tübingen, Auf der Morgenstelle 14, \\ D-72076 Tübingen, Germany \\ ${ }^{2}$ Laboratoire de Physique Théorique des Particules Élémentaires, \\ Université P. et M. Curie, 4 Place Jussieu, 75252 Paris Cedex 05, France
}

\begin{abstract}
We investigate the hadronic two-body decay modes of the scalar-isoscalar $f_{0}(1370), f_{0}(1500)$ and $f_{0}(1710)$ states as resulting from the mixture of the lowest lying scalar glueball with the isoscalar states of the ground state ${ }^{3} P_{0}$ $Q \bar{Q}$ nonet. In the decay analysis we take into account the direct coupling of the quarkonia and glueball components of the $f_{0}$ states to the quarkonia components of the two-meson final state with the decay dynamics inspired by the strong coupling limit of QCD. We calculate partial decay widths for the $f_{0}$ states in the proposed three-state mixing schemes and discuss their compatibility with the observed decay features. Finally, we determine the glueball-quarkonia content of the $f_{0}$ states from a detailed fit to experimental decay data of $f_{0}(1500)$ and give predictions for the partial decay widths of $f_{0}(1370)$ and $f_{0}(1710)$, providing thus a sensitive test of the proposed mixing scheme.
\end{abstract}

Keywords : scalar glueball, hadronic decay of mesons, meson spectroscopy 13.25.Jx, 12.39.Mk, 1440.Cs 


\section{INTRODUCTION}

The identification of mesons beyond the conventional valence quark-antiquark $(Q \bar{Q})$ pair configuration is one of the most challenging tasks in low-energy meson spectroscopy.

For instance, as a consequence of gluon self-couplings in QCD the usual $Q \bar{Q}$ spectrum is expected to be supplemented by mesonic states with pure gluonic composition, that is glueballs [1].

Lattice QCD, in the quenched approximation, predicts the lightest glueball state $\left(G_{0}\right)$ to be a scalar $\left(J^{P C}=0^{++}\right)$lying in the mass range of $1.4-1.8 \mathrm{GeV}$ [2].3]. Since the observed isovector and isodoublet states $a_{0}(1450)$ and $K_{0}(1430)$ set the natural mass scale of the

ground state scalar meson nonet, the isoscalar $Q \bar{Q}$ partners $n \bar{n} \equiv \frac{1}{\sqrt{2}}(u \bar{u}+d \bar{d})$ and $s \bar{s}$ are expected to lie in a mass region close to the predicted ground state glueball. The vicinity of masses for the pure $G_{0}, n \bar{n}$ and $s \bar{s}$ mesonic states suggests that the intrusion of the scalar glueball into the scalar $Q \bar{Q}$ meson spectrum is accompanied by significant mixing of $G_{0}$ with the isoscalar members of the ${ }^{3} P_{0}$ nonet [4,5].

Several glueball-quarkonia mixing schemes [4 7] have been proposed to account for this scenario and to reveal the $G_{0} / Q \bar{Q}$ nature of the scalar-isoscalar states $f_{0}(1370), f_{0}(1500)$, established by Crystal Barrel at LEAR [8] in proton-antiproton annihilation reactions, and the $f_{0}(1710)$ [9], which is the scalar component of the $f_{j=0,2}(1710)$ [10]. In a simplified picture considered in Refs. [4,5] the scalar glueball ground state $G_{0}$ mixes with the lowest lying ${ }^{3} P_{0}$ isoscalar quarkonium states. Further admixture of the first radially excited ${ }^{3} P_{0}$ nonet is introduced in Refs. [6,7]. However, the restriction to the glueball and near lying ${ }^{3} P_{0}$ quarkonia ground states is a good approximation provided that the excited glueball and quarkonia states are high enough in mass.

Quantitative predictions in the three-state mixing schemes of Refs. [4, 5] for the glueballquarkonia content of $f_{0}(1370), f_{0}(1500)$ and $f_{0}(1710)$ differ substantially due to orthogonal theoretical assumptions concerning the level ordering of the bare states before mixing. While Ref. [4] uses for the input masses of the bare states $M(n \bar{n})<M\left(G_{0}\right)<M(s \bar{s})$, in Ref. [5] 
the pure glueball lies above the scalar $Q \bar{Q}$ state. The difference of level ordering in the input bare masses leads to a substantially different $G_{0} / Q \bar{Q}$ content of the physical $f_{0}$ states, especially for $f_{0}(1500)$ and $f_{0}(1710)$. While in Ref. [4], the glueball is distributed with nearly equal strength over the three $f_{0}$ states and $f_{0}(1710)$ has a large $s \bar{s}$ component, the analysis of Ref. [5] leads to a dominant $G_{0}$ structure in the $f_{0}(1710)$, while $f_{0}(1500)$ is a nearly pure $s \bar{s}$ state. The results for the $f_{0}(1370)$ are comparable in both approaches, with a large $n \bar{n}$ component residing in this state. The observed total width of the $f_{0}(1370)$ [ 8 and its strong coupling to the $\pi \pi$ decay channel is in good agreement with naive quark model expectations 11,12 for a $n \bar{n}{ }^{3} P_{0}$ state and seems to confirm its dominant $n \bar{n}$ structure. Therefore, the main difference between the two mixing schemes is the amount of glueball and $s \bar{s}$ strength residing in the $f_{0}(1500)$ and $f_{0}(1710)$ states, respectively. A further test of the proposed mixing schemes, where the glueball ground state intrudes in the scalar quarkonium sector, is the analysis of the decay modes of the observed $f_{0}$ states. The decay of $f_{0}(1500)$ into two pseudoscalar mesons was studied in leading order of strong coupling QCD [4]. Neglecting possible gluonic components of the isoscalar mesons in the final state, the $\pi \pi, K \bar{K}, \eta \eta$ and $\eta \eta^{\prime}$ decay modes are entirely driven by the quarkonia components of the $f_{0}(1500)$ state. In this approximation, results for the partial decay widths deduced from $S U(3)$ flavor symmetric couplings are quite similar for both mixing schemes and cannot be discriminated [4], e.g. the observed weak $K \bar{K}$ decay mode is obtained in both schemes by the destructive interference between the $n \bar{n}$ and $s \bar{s}$ components.

In order to reveal the difference of the proposed three state mixing schemes, one should take into account the direct coupling of the $G_{0}$ component to the final state mesons. This mechanism occurs in the leading of strong coupling QCD [4] by the transition $G_{0} \rightarrow G_{0} G_{0}$, which can produce final states with isoscalar mesons $\left(\eta \eta, \eta \eta^{\prime}\right.$ and $\sigma \sigma$, where $\sigma$ is the broad low lying $\pi \pi$ S-wave resonance) with possibly a sizable gluonic component. Independent of a possible small glueball component in the final state isoscalars is the decay process $G_{0} \rightarrow(Q \bar{Q})(Q \bar{Q})$. This next-to-leading order transition can contribute to all two-body decay modes of the mixed $f_{0}$ states. An investigation [13 of the two-body decay modes $\sigma \sigma, \rho \rho$ and $\pi^{*}(1300) \pi$ 
of the $f_{0}(1500)$, all leading to the $4 \pi$ decay channel, indicates the possible presence of a sizable direct coupling of the gluonic component of the $f_{0}(1500)$ to the $4 \pi$ decay channels. Neglecting a possible $G_{0}$ component in $\sigma$, the leading order decay mechanism predicts [13] the hierarchy of branching ratios $\mathrm{BR}$ with $B R(\rho \rho)_{\sim}^{>B R}(\pi \pi)_{\sim}^{>} B R(\sigma \sigma)>B R\left(\pi^{*}(1300) \pi\right)$, independent of any particular three-state mixing scheme. The results are in strong conflict with current Crystal Barrel data, where the $\rho \rho$ decay mode of the $f_{0}(1500)$ is weak or even absent [15] and a stronger coupling to the $4 \pi$ modes than to the $2 \pi$ decay channel [8] is required.

In the present work, going beyond the lowest order decay mechanism, we couple the $Q \bar{Q}$ components of the mixed $f_{0}$ states to the quarkonia components of the final state mesons. In the decay analysis we also take into account the transition $G_{0} \rightarrow(Q \bar{Q})(Q \bar{Q})$ occurring as a next-to-leading order decay mechanism deduced from strong coupling QCD 四. Inclusion of the direct coupling of the $G_{0}$ component of the mixed $f_{0}$ states to the quarkonia content of the final state mesons will lead to contradictory physical consequences in the proposed threestate mixing schemes of Refs. [四,5], in particular for the physical state $f_{0}(1710)$. Because of the shortcomings of these proposed mixing models, we undertake to extract a three-state mixing scheme based on a fit to the detailed experimental data of $f_{0}(1500)$ two-body decays and give the predictions for partial decay widths of the partner states $f_{0}(1370)$ and $f_{0}(1710)$. This paper is organised as follows. In Sec. 【1 we introduce the three-state mixing scheme of the lowest lying scalar glueball with the quarkonia states and the decay dynamics as suggested by the strong coupling limit of QCD. In Sec. III we develop the formalism for the decay of the $f_{0}$ states by its $Q \bar{Q}$ components in the framework of the pair creation or ${ }^{3} P_{0}$ model. In Sec. $\mathbb{V}$ we construct a digluonium wave function based on cavity QCD for the effective description of the scalar glueball state. Furthermore, we indicate the transition mechanism for the decay of the $G_{0}$ component into $(Q \bar{Q})(Q \bar{Q})$. The results are presented in Sec. $\nabla$; we first show how distinct decay patterns result from the unmixed $Q \bar{Q}$ and $G_{0}$ components of the $f_{0}$ states. We then give partial widths for the two-body decay modes of the $f_{0}$ states in the mixing schemes of Refs. [4,5] and confront the results with experiment. 
Finally, we present a three-state mixing scheme deduced from a detailed fit to the experimental data for $f_{0}(1500)$ decays and give predictions for the partial decay widths of $f_{0}(1370)$ and $f_{0}(1710)$, providing thus a sensitive test of the proposed mixing scheme. A summary and conclusions are given in Sec. V1. 


\section{THREE-STATE MIXING AND DECAY OF SCALAR-ISOSCALAR STATES IN STRONG COUPLING QCD}

In the lattice formulation of the strong coupling limit $(g \rightarrow \infty)$ of QCD [4],11], glueballs and $Q \bar{Q}$ mesons are noninteracting eigenstates of the QCD Hamiltonian. Departure from this limit causes mixing and decay of the strong coupling eigenstates [4, [1]].

To lowest order, the mixing of the scalar glueball $G_{0}$ and the ${ }^{3} P_{0}$ quarkonia states $n \bar{n}=$ $1 / \sqrt{(2)}(u \bar{u}+d \bar{d})$ and $s \bar{s}$ can be introduced by the interaction Hamiltonian $H_{I}$ [5]

$$
\left(H_{I}\right)=\left(\begin{array}{ccc}
m_{G_{0}} & z & \sqrt{2} z \\
z & m_{s \bar{s}} & 0 \\
\sqrt{2} z & 0 & m_{n \bar{n}}
\end{array}\right),
$$

where $z=<G_{0}\left|H_{I}\right| s \bar{s}>=<G_{0}\left|H_{I}\right| n \bar{n}>/ \sqrt{2}$ represents the flavor independent mixing strength between the glueball and quarkonium states. The masses of the bare states $i=$ $G_{0}, s \bar{s}, n \bar{n}$ before mixing are denoted by $m_{i}$. Deviation from the $S U(3)$ flavor symmetry is known to be small [14] and quarkonia mixing is assumed to be a higher order perturbation in the strong coupling eigenstates $\left|G_{0}>,\right| s \bar{s}>$ and $\mid n \bar{n}>$ 幽. All parameters of $H_{I}$ can be taken to be real and positive.

$H_{I}$ possesses three eigenstates $\mid f_{0}(M)>$ with physical masses $\mathrm{M}$, which are are given by the linear combinations:

$$
\left|f_{0}(M)>=a_{n \bar{n}}\right| n \bar{n}>+a_{s \bar{s}}\left|s \bar{s}>+a_{G_{0}}\right| G_{0}>
$$

with $a_{n \bar{n}}^{2}+a_{s \bar{s}}^{2}+a_{G_{0}}^{2}=1$. Choosing the bare mass $m_{n \bar{n}}$ to be equal to that of the observed isovector state $a_{0}(1450)$ [10], the remaining parameters in $H_{I}$ can be adjusted to give the masses of the observed resonances $f_{0}(1370), f_{0}(1500)$ and $f_{0}(1710)$, which fixes the quarkonia-glueball composition of the physical states [5]. In Ref. [4] it is assumed that the level spacings of the bare states fulfil the relation $\left(m_{G_{0}}-m_{n \bar{n}}\right) / 2=m_{s \bar{s}}-m_{G_{0}}=z$, which again determines the state vector of the physical $f_{0}(M)$ states. In this case $f_{0}(1500)$ is degenerate in mass with the $G_{0}$ state and the physical masses $\mathrm{M}$ of the mixing partners 
$f_{0}(1370)$ and $f_{0}(1710)$ depend on the mixing strength z.

However, there are sizable uncertainties in these procedures, particularly since the mass of the $f_{0}(1370)$ is poorly determined, ranging from 1200 - $1500 \mathrm{MeV}$ [10 and the exact level spacing of the bare states in unknown. For these reasons we will also present an alternative strategy by assuming that $f_{0}(1500)$ is one of the eigenstates of $H_{I}$ and by extracting the mixing coefficients $a_{i}\left(i=n \bar{n}, s \bar{s}, G_{0}\right)$ directly from the experimental two-body decay data. This will allow us to give the bare masses $m_{i}$ entering in $H_{I}$ of Eq. (1), in terms of the mixing strength $\mathrm{z}$, which in turn is restricted by the physical masses of the partners of the $f_{0}(1500)$. The resulting glueball-quarkonia composition of these partner states, however, is completely independent of $\mathrm{z}$, solely determined by the mixing coefficients of the $f_{0}(1500)$.

In the minimal constituent picture $G_{0}$ consists of two gluons bound in a color singlet configuration with $J^{P C}=0^{++}$. For finite $g$ the mixed $\mid f_{0}(M)>$ decays in lowest order by its $G_{0}$ and $Q \bar{Q}$ components. The relevant diagrams are shown in Figs. 1 and 2. The transition $G_{0} \rightarrow G_{0} G_{0}$ of Fig. 1 couples the $G_{0}$ component of the physical state $\mid f_{0}(M)>$ to the $G_{0}$ component of the final state isoscalar mesons. The constituent gluons of the initial state decay into two pairs of low energy gluons which involves two three-gluon vertices in the decay mechanism. We will neglect this transition in our decay analysis by assuming that the considered final state isoscalar mesons do not contain a $G_{0}$ component. In principle, the transition of Fig. 1 can contribute to the decay modes $f_{0}(M) \rightarrow \eta \eta, \eta \eta^{\prime}$ and $\sigma \sigma$, depending on the unknown overlap of the $\eta, \eta^{\prime}$ and $\sigma$ with the corresponding glueball states. Current evidence for the relevance of such a process involving the pseudoscalars $\eta$ and $\eta^{\prime}$ is not compelling, as for example discussed in Ref. «1. For the scalar $\sigma$ meson a large coupling to the glueball state is not excluded [4, 10]. Therefore, we should keep in mind that the $G_{0} \rightarrow G_{0} G_{0}$ transition could induce some changes for the $\eta \eta, \eta \eta^{\prime}$ and $\sigma \sigma$ decay modes of the $f_{0}$ states.

The leading order decay mechanism $Q \bar{Q} \rightarrow(Q \bar{Q})(Q \bar{Q})$, illustrated in Fig. 2, leads to the decay of $\mid f_{0}(M)>$ by its quarkonia components $Q \bar{Q}$ and is familiar from the OZI-allowed meson decay 11, 13, 16. This process will be described in Sec. III in the framework of the 
${ }^{3} P_{0}$ pair creation model [17.

Here we also take into account the next to leading order decay mechanism $G_{0} \rightarrow(Q \bar{Q})(Q \bar{Q})$ as indicated in Fig. 3. In this mechanism the $G_{0}$ component of the physical $f_{0}(M)$ states couples to the final state $Q \bar{Q}$ mesons by conversion of the constituent gluons into quarkantiquark pairs. Sec. $\mathbb{\Pi}$ is devoted to the description of this process.

Neglecting a possible $G_{0}$ component in the final state isoscalar mesons, the partial decay width $\Gamma_{f_{0} \rightarrow B C}$ for the decay of $\mid f_{0}(M)>$ into a two-meson state B $\mathrm{C}$ can be written as

$$
\begin{aligned}
\Gamma_{f_{0} \rightarrow B C} & =2 \pi K \frac{E_{B} E_{C}}{M_{f_{0}}} \sum_{l_{B C}} \int d \Omega_{K}\left|T_{f_{0} \rightarrow B C}^{\left(l_{B C}\right)}\right|^{2} \\
& =\sum_{l_{B C}}\left|\lambda M_{Q \bar{Q} \rightarrow B C}^{\left(l_{B C}\right)}+\gamma^{2} M_{G_{0} \rightarrow B C}^{\left(l_{B C}\right)}\right|^{2}
\end{aligned}
$$

where $M_{Q \bar{Q} \rightarrow B C}^{\left(l_{B C}\right)}$ and $M_{G_{0} \rightarrow B C}^{\left(l_{B C}\right)}$ denote the transition amplitudes including phase space of the respective decay mechanism. The sum extends over the relative orbital angular momentum $l_{B C}$ between the mesons $\mathrm{B}$ and $\mathrm{C}$. With the decay momentum $|\vec{K}|=K, E_{i}=\sqrt{M_{i}^{2}+\vec{K}^{2}}$ is the energy of the final state mesons $i=B, C$ with mass $M_{i}$. The strengths of the quarkgluon coupling and the pair creation amplitude in the ${ }^{3} P_{0}$ model are factorised out and denoted by $\gamma$ and $\lambda$, respectively. The relative phase between the two transition amplitudes is fixed by choosing $M_{Q \bar{Q} \rightarrow B C}^{\left(l_{B C}\right)}$ and $M_{G_{0} \rightarrow B C}^{\left(l_{B C}\right)}$ to be real and the ratio $\gamma^{2} / \lambda$ to be complex with

$$
\frac{\gamma^{2}}{\lambda} \equiv \kappa e^{i \phi}
$$

In this case $\Gamma_{f_{0} \rightarrow B C}$ is given by

$$
\Gamma_{f_{0} \rightarrow B C}=\lambda^{2}\left\{M_{Q \bar{Q} \rightarrow B C}^{2}+\kappa^{2} M_{G_{0} \rightarrow B C}^{2}+2 \kappa \cos \phi \sum_{l_{B C}} M_{Q \bar{Q} \rightarrow B C}^{\left(l_{B C}\right)} M_{G_{0} \rightarrow B C}^{\left(l_{B C}\right)}\right\} .
$$

with the summation over $l_{B C}$ included in the definition of $M_{Q \bar{Q}\left(G_{0}\right) \rightarrow B C}^{2}$. Finally, in order to take into account properly the available phase space for the decay $f_{0} \rightarrow B C$ we average over the mass spectrum $f(m)$ of broad mesons:

$$
\begin{aligned}
\Gamma_{f_{0} \rightarrow B C} & =\int d m_{f_{0}} d m_{B} d m_{C} \Gamma_{f_{0} \rightarrow B C}\left(m_{f_{0}}, m_{B}, m_{C}\right) f\left(m_{f_{0}}\right) f\left(m_{B}\right) f\left(m_{C}\right) \\
f(m) & \propto \frac{\left(\Gamma_{i} / 2\right)^{2}}{\left(m-M_{i}\right)^{2}+\left(\Gamma_{i} / 2\right)^{2}}
\end{aligned}
$$


with a threshold cutoff as in Ref. [18]. The individual masses $M_{i}$ and widths $\Gamma_{i}$ of the resonances are taken from the Particle Data Group.

The mass distribution of the scalar-isoscalar $\sigma$ meson is parameterised with $M_{\sigma}=760 \mathrm{MeV}$ and $\Gamma_{\sigma}=640 \mathrm{MeV}$ [19]. For the $\pi^{*}(1300)$ we use the resonance width of $\Gamma_{\pi^{*}(1300)}=200 \mathrm{MeV}$ [20]. The relationship between $f_{0}(1370)$ and the broad structure around $1100 \mathrm{MeV}$ [8] called $f_{0}(400-1200)$ [10] is not entirely clear. If the two states are manifestations of a single object, then $\Gamma_{f_{0}(1370)} \approx 700 \mathrm{MeV}[\mathbb{B}]$. For two independent states one has $\Gamma_{f_{0}(1370)}=$ $351 \pm 41 \mathrm{MeV}, M_{f_{0}(1370)}=1360 \pm 23 \mathrm{MeV}$ [ \&] and $\Gamma_{f_{0}(1370)}=230 \pm 15 \mathrm{MeV}, M_{f_{0}(1370)}=$ $1300 \pm 15 \mathrm{MeV}$ [28]. 


\section{DECAY OF THE QUARKONIA COMPONENTS}

The decay of the quarkonia components of the $\mid f_{0}(M)>$ states into the final state mesons $\mathrm{B}$ and $\mathrm{C}$ is calculated in the non-relativistic ${ }^{3} P_{0}$ or pair creation model [17]. This model has a solid foundation in strong coupling QCD [11,21,22]. It describes the OZI-allowed decay $Q \bar{Q} \rightarrow B C$ by the creation of a $Q \bar{Q}$ pair with quantum numbers $\left(I^{G}\left(J^{P C}\right)=0^{+}\left(0^{++}\right)\right)$out of the hadronic vacuum, as indicated in Fig. 2. In the evaluation one also has to consider a second diagram where the quark 3 (4) goes into the meson $\mathrm{C}(\mathrm{B})$. The constituent quarks of the initial state are spectators in the transition. The strength of this transition is governed by the dimensionless constant $\lambda$, which is related to the pair creation probability. From detailed fits to tensor meson decay [4,23] it is concluded that the pair creation mechanism is flavor independent.

The nonperturbative $Q \bar{Q}{ }^{3} P_{0}$ vertex can be defined as

$$
V_{3_{0}}^{(43)}=\lambda \delta\left(\vec{p}_{4}+\vec{p}_{3}\right)\left[\mathcal{Y}_{1 \mu}^{*}\left(\vec{p}_{4}-\vec{p}_{3}\right) \otimes \sigma_{-\mu}^{(43) \dagger}\right]_{00} \mathbf{1}_{F}^{(43)} \mathbf{1}_{C}^{(43)}
$$

where $\vec{p}_{4(3)}$ are the momenta of the quark (antiquark) $4(3)$ and $\mathcal{Y}_{1}(\vec{p})=|\vec{p}| Y_{1}(\hat{p})$. The identity operators $\mathbf{1}_{\mathbf{F}}$ and $\mathbf{1}_{\mathbf{C}}$ project onto singlet states in flavor $(\mathrm{F})$ and color $(\mathrm{C})$ space of the created $Q \bar{Q}$ pair (43).

Using the harmonic oscillator ansatz $\Psi_{n=0, l=1}\left(\overrightarrow{p_{1}}, \overrightarrow{p_{2}}\right)$, the wave function of the $Q \bar{Q}$ component of the mixed $f_{0}$ can be expressed in its centre-of-momentum frame as:

$$
\begin{aligned}
\Psi_{f_{0}}\left(\vec{p}_{1}, \vec{p}_{2}\right)= & \delta\left(\vec{p}_{1}+\vec{p}_{2}\right)\left[\chi_{S=1}(12) \otimes \Psi_{n=0, l=1}\left(\vec{p}_{1}, \vec{p}_{2}\right)\right]_{J=0} \chi_{F}^{f_{0}}(12) \chi_{C}^{(12)} \\
= & {\left[\frac{2 R_{Q \bar{Q}}^{5}}{3 \sqrt{\pi}}\right]^{\frac{1}{2}} \delta\left(\vec{p}_{1}+\vec{p}_{2}\right) \exp \left\{-\frac{1}{8} R_{Q \bar{Q}}^{2}\left(\vec{p}_{1}-\vec{p}_{2}\right)^{2}\right\} } \\
& \cdot\left[\chi_{S=1}(12) \otimes \mathcal{Y}_{l=1}\left(\vec{p}_{1}-\vec{p}_{2}\right)\right]_{J=0} \chi_{F}^{f_{0}}(12) \chi_{C}^{(12)}
\end{aligned}
$$

where $R_{Q \bar{Q}}$ is the size parameter for the $Q \bar{Q}$ component. The intrinsic spin $\chi_{S=1}(12)$ of the $Q \bar{Q}$ pair (12) is coupled with relative angular momentum $l=1$ to the $J^{P C}=0^{++}$scalar ground state $(n=0)$ and $\chi_{C}^{(12)}$ is the color singlet wave function. The $S U(3)$ flavor part $\chi_{F}^{f_{0}}(12)$ is given by 


$$
\chi_{F}^{f_{0}}(12)=a_{n \bar{n}}\left|n_{1} \bar{n}_{2}>+a_{s \bar{s}}\right| s_{1} \bar{s}_{2}>
$$

with the quarkonia mixing coefficients $a_{i}(i=n \bar{n}, s \bar{s})$ of the three-state mixing scheme introduced in Sec. ㅍ. For the final state mesons $i(i=B, C)$ with momentum $\vec{P}_{i}$, total spin $J_{i}$, relative orbital angular momentum $l_{i}$ and intrinsic spin $S_{i}$ we use the wave functions

$$
\Psi_{i}\left(\vec{p}_{l}, \vec{p}_{m}\right)=\delta\left(\vec{p}_{l}+\vec{p}_{m}-\vec{P}_{i}\right)\left[\chi_{S_{i}}(l m) \otimes \Psi_{n_{i} l_{i}}\left(\overrightarrow{p_{l}}, \overrightarrow{p_{m}}\right)\right]_{J_{i}} \chi_{F}^{i}(l m) \chi_{C}^{(l m)}
$$

where $\vec{p}_{l(m)}$ are internal quark momenta for the quarks $l(m)$. Explicit forms for the harmonic oscillator wave functions $\Psi_{n_{i} l_{i}}$ used can be found in Appendix A.

The transition amplitude $M_{Q \bar{Q} \rightarrow B C}^{\left(l_{B C}\right)}$, as defined by Eq. (3), separates into a flavor part $M_{Q \bar{Q} \rightarrow B C}^{F}$

$$
M_{Q \bar{Q} \rightarrow B C}^{F}=<\chi_{F}^{B}(13) \chi_{F}^{C}(42)\left|\mathbf{1}_{F}^{(43)}\right| \chi_{F}^{f_{0}}(12)>
$$

and the spin-spatial-color part $M_{Q \bar{Q} \rightarrow B C}^{\left(l_{B C}\right) S S C}$

$$
\begin{aligned}
M_{Q \bar{Q} \rightarrow B C}^{\left(l_{B C}\right) S S C}= & \sqrt{2 \pi K} \sqrt{\frac{E_{B} E_{C}}{M_{f_{0}}}} \frac{1}{\sqrt{3}} \int \prod_{i=1, \ldots 4} d \overrightarrow{p_{i}} \delta\left(\overrightarrow{p_{4}}+\overrightarrow{p_{3}}\right) \delta\left(\overrightarrow{p_{1}}+\overrightarrow{p_{2}}\right) \\
& \delta\left(\overrightarrow{p_{1}}+\overrightarrow{p_{3}}-\vec{K}\right) \delta\left(\overrightarrow{p_{2}}+\overrightarrow{p_{4}}+\vec{K}\right)\left[\chi_{S_{B}}^{(13)} \otimes \Psi_{n_{B} l_{B}}\left(\overrightarrow{p_{1}}, \overrightarrow{p_{3}}\right)\right]^{\dagger} \\
& {\left[\chi_{S_{C}}^{(42)} \otimes \Psi_{n_{C} l_{C}}\left(\overrightarrow{p_{4}}, \overrightarrow{p_{2}}\right)\right]^{\dagger}\left[\mathcal{Y}_{1 \mu}^{*}\left(\overrightarrow{p_{4}}-\overrightarrow{p_{3}}\right) \otimes \sigma_{-\mu}^{(43) \dagger}\right]_{00}\left[\chi_{S=1}^{(12)} \otimes \Psi_{n=0 l=1}\left(\overrightarrow{p_{1}}, \overrightarrow{p_{2}}\right)\right]_{J=0} }
\end{aligned}
$$

with the decay momentum $\vec{K}$ and the color matrix element $\left\langle\chi_{C}^{(13)} \chi_{C}^{(42)}\left|\mathbf{1}_{C}\right| \chi_{C}^{(12)}>=\sqrt{1 / 3}\right.$. The amplitude $M_{Q \bar{Q} \rightarrow B C}^{\left(l_{B C}\right) S S C}$ is evaluated analytically. Since there exists exhaustive literature 11, 16, 17 on the ${ }^{3} P_{0}$ model we just cite the results in Appendix B. Values for the various flavor matrix elements are given in Table凹. The results of Table凹were obtained by assuming $\chi_{F}^{\omega}=\mid n \bar{n}>$. Thereby we have used the following definitions for the flavor content of the $\eta$ and $\eta^{\prime}$ mesons:

$$
\begin{aligned}
& \psi_{F}^{\eta}=\alpha_{P S}\left|n \bar{n}>-\beta_{P S}\right| s \bar{s}> \\
& \psi_{F}^{\eta^{\prime}}=\beta_{P S}\left|n \bar{s}>\quad+\alpha_{P S}\right| s \bar{s}>.
\end{aligned}
$$

The quantities $\alpha_{P S}$ and $\beta_{P S}$ are simply related to the pseudoscalar mixing angle $\theta_{P}$ as defined by the Particle Data Group [10]. For numerical results we use the value of $\theta_{P}=-17.3^{\circ}$ 
24] or equivalently $\alpha_{P S}=0.794, \beta_{P S}=0.608$.

The partial decay widths for the quarkonia decay $Q \bar{Q} \rightarrow B C$ are defined as in Eq. (5) and depend on the pair creation amplitude $\lambda$ and the quarkonia size parameters $R_{Q \bar{Q}}$ and $R_{B}=R_{C}$ for the decay channels considered here.

Exhaustive experimental data exist for the hadronic decays of the ground state tensor meson nonet and the strategy is to extract the meson size parameters combined with the ${ }^{3} P_{0}$ strength from a full scale decay analysis. A recent fit to tensor meson decays 23 yields $\lambda^{2}=2.11$ and $R_{B}=3.65 \mathrm{GeV}^{-1}$ for nonstrange S-wave mesons and strange tensor mesons, suggesting $R_{a_{1}}=R_{Q \bar{Q}}=3.65 \mathrm{GeV}^{-1}$. S-wave mesons containing strange quarks have an effectively reduced size with $R_{B}=2.48 \mathrm{GeV}^{-1}$ for $\eta$ and $\eta^{\prime}$ and $R_{K}=2.82 \mathrm{GeV}^{-1}$ for $\mathrm{K}$. No flavor symmetry breaking at the ${ }^{3} P_{0}$ vertex was obtained, hence $\lambda$ is independent of the created $Q \bar{Q}$ flavor. Due to orthogonality of the $\pi$ and $\pi^{*}(1300)$ wave functions the respective size parameters fulfil the relation $R_{\pi}=R_{\pi^{*}}$. The $R_{\sigma}$ dependence of the quarkonia decay amplitude was shown to be small [13]. We therefore choose $R_{\sigma}=R_{\pi}$ for our numerical results presented in the forthcoming section. 


\section{DECAY OF THE GLUEBALL COMPONENT}

In this section we consider the two-body decay of the ground state scalar glueball $\left(G_{0}\right)$ in the next-to-leading order decay mechanism of strong coupling QCD [4]. For the decay analysis we adopt a non-relativistic picture where $G_{0}$ is a bound state of two massive constituent gluons g. The decay is proceeding via the conversion of the constituent gluons into $Q \bar{Q}$ pairs as illustrated in Fig. 3. In the evaluation a second diagram with different quark rearrangement in the final state analogous to quarkonia decay has also to be considered. The elementary quark-gluon interaction vertex is given to lowest order in the non-relativistic limit by

$$
V^{\left(g_{i} Q_{l} \bar{Q}_{k}\right)}=\gamma \delta\left(\vec{q}_{i}-\vec{p}_{l}-\vec{p}_{k}\right)\left(\vec{\sigma}_{(l k)} \cdot \vec{\epsilon}_{i}\right) \mathbf{1}_{F}^{(l k)}\left(\frac{1}{2} \sum_{a=1}^{8} \lambda_{(l k)}^{a} A_{i}^{a}\right)
$$

with the internal momenta $\vec{q}_{i}$ for gluon $\mathrm{i}=1,2$ and $\vec{p}_{l(k)}$ for the quarks with label $l=1,3(\mathrm{k}=2,4)$.

The identity operator $\mathbf{1}_{F}^{(l k)}$ projects onto a flavor singlet state of the created $Q \bar{Q}$ pair (lk). The last term in Eq. (15) is the color part of the interaction vertex with the Gell-Mann matrices $\lambda^{a}$ acting in color space of $(\mathrm{lk})$. The color octet wave function of the gluon i with polarisation vector $\vec{\epsilon}_{i}$ is denoted by $A_{i}^{a}$. The strength of the $g_{i} \rightarrow Q_{l} \bar{Q}_{k}$ transition is given by $\gamma$. We assume that flavor symmetry is manifested in the quark-gluon coupling [⿴囗十], hence $<n \bar{n}|V| g>\equiv<s \bar{s}|V| g>=\gamma$. In order to evaluate the decay amplitude $M_{G_{0} \rightarrow B C}$ we will construct a non-relativistic digluonium wave function from cavity QCD.

\section{A. Digluonium wave function}

In constructing the glueball wave function we use the Bag Model as a guidance. The ground state scalar glueball is realized in the Bag Model by the coupling of two transverse electric (TE) modes of the constituent gluon $\left(J^{P C}=1^{+-}\right)$to scalar quantum numbers $J^{P C}=0^{++}$25]. Using the non-relativistic TE gluon mode, proposed in Ref. [26], the scalar glueball wave function is in simple correspondence with the Bag Model solution and can be written as 


$$
\begin{aligned}
\Psi_{G_{0}}\left(\vec{r}_{1}, \vec{r}_{2}\right) & =\left[T E_{1}\left(1^{+-}\right) \otimes T E_{2}\left(1^{+-}\right)\right]_{J=0} \\
& =\left\{\left[\Psi_{n_{1}=0 l_{1}=1}\left(\vec{r}_{1}\right) \otimes \epsilon_{1}^{\mu}\right]_{1} \otimes\left[\Psi_{n_{2}=0 l_{2}=1}\left(\vec{r}_{2}\right) \otimes \epsilon_{2}^{\mu^{\prime}}\right]_{1}\right\}_{J=0} \Psi_{C}^{(12)}
\end{aligned}
$$

where $\Psi_{n=0 l=1}(\vec{r})$ is the single particle harmonic oscillator wave functions as defined in Appendix A. The spherical components of the gluon polarisation vector $\vec{\epsilon}$ are given by $\epsilon^{\mu\left(\mu^{\prime}\right)}$. The color singlet wave function $\Psi_{C}^{(12)}$ for the two gluon state is

$$
\Psi_{C}^{(12)}=\frac{1}{\sqrt{8}} \sum_{b=1}^{8} A_{1}^{b} A_{2}^{b}
$$

where we have chosen the "cartesian" basis $A^{b}$ with

$$
A^{b}=\frac{1}{\sqrt{2}} \sum_{i j=1}^{3}\left(\lambda_{i j}\right)^{b} c_{i} \bar{c}_{j}
$$

for the octet representation of color $S U(3)$ with $c_{i}$ and $\bar{c}_{j}$ being the basis states for the fundamental representations $\mathbf{3}$ and $\overline{\mathbf{3}}$.

$\Psi_{G_{0}}\left(\vec{r}_{1}, \vec{r}_{2}\right)$ can be recoupled as

$$
\begin{aligned}
\Psi_{G_{0}}\left(\vec{r}_{1}, \vec{r}_{2}\right)= & \sqrt{3} \sum_{g}(-)^{g+1}\left\{\begin{array}{ccc}
1 & 1 & 1 \\
1 & 1 & g
\end{array}\right\} \sqrt{2 g+1} \\
& \cdot\left\{\left[\Psi_{n_{1}=0 l_{1}=1}\left(\vec{r}_{1}\right) \otimes \Psi_{n_{2}=0 l_{2}=1}\left(\vec{r}_{2}\right)\right]_{g} \otimes\left[\epsilon_{1}^{\mu} \otimes \epsilon_{2}^{\mu^{\prime}}\right]_{g}\right\}_{J=0} \Psi_{C}^{(12)} .
\end{aligned}
$$

Introducing relative and centre-of-mass coordinates $\vec{r}=\vec{r}_{1}-\vec{r}_{2}$ and $\vec{R}=1 / 2\left(\vec{r}_{1}+\vec{r}_{2}\right)$, respectively, we perform the expansion 27]

$$
\begin{gathered}
{\left[\Psi_{n_{1}=0 l_{1}=1}\left(\vec{r}_{1}\right) \otimes \Psi_{n_{2}=0 l_{2}=1}\left(\vec{r}_{2}\right)\right]_{g}=} \\
\sum_{n l N L}<n l, N L, g \mid n_{1}=0 l_{1}=1, n_{2}=0 l_{2}=1, g> \\
{\left[\Psi_{n l}(\vec{r}) \otimes \Psi_{N L}(\vec{R})\right]_{g}}
\end{gathered}
$$

where energy conservation restricts the sum to $2 n+l+2 N+L=2$. The relevant values for the transformation brackets $<n l, N L, g \mid n_{1} l_{1}, n_{2} l_{2}, g>$ can be found in Appendix C.

When projecting out the spurious centre-of-mass excitations, the normalised glueball wave function can be written in its centre-of-momentum frame as

$$
\begin{aligned}
\Psi_{G_{0}}\left(\vec{q}_{1}, \vec{q}_{2}\right)= & \left(\sqrt{\frac{5}{9}}\left\{\Psi_{n=0 l=2}\left(\vec{q}_{1}, \vec{q}_{2}\right) \otimes\left[\epsilon_{1}^{\mu} \otimes \epsilon_{2}^{\mu^{\prime}}\right]_{2}\right\}_{0}\right. \\
& \left.-\frac{2}{3}\left\{\Psi_{n=1 l=0}\left(\vec{q}_{1}, \vec{q}_{2}\right) \otimes\left[\epsilon_{1}^{\mu} \otimes \epsilon_{2}^{\mu^{\prime}}\right]_{0}\right\}_{0}\right) \delta\left(\vec{q}_{1}+\vec{q}_{2}\right) \Psi_{C}^{(12)}
\end{aligned}
$$


with the internal gluon momenta $\vec{q}_{i}(i=1,2)$ and $\Psi_{n l}\left(\vec{q}_{1}, \vec{q}_{2}\right)$ given in Appendix A.

\section{B. Digluonium decay amplitudes}

With the digluonium wave function of Eq. (21) were are now in the position to evaluate the decay amplitudes for the process $G_{0} \rightarrow B C$ of Fig. 3. The decay amplitude $M_{G_{0} \rightarrow B C}^{\left(l_{B C}\right)}$, as defined by Eq. (3), separates in analogy to quarkonia decay into a flavor part $M_{G_{0} \rightarrow B C}^{F}$ with

$$
M_{G_{0} \rightarrow B C}^{F}=<\chi_{F}^{B}(13) \chi_{F}^{C}(42)\left|\mathbf{1}_{F}^{(12)} \mathbf{1}_{F}^{(43)}\right| 0>
$$

and a spin-spatial-color part

$$
\begin{aligned}
M_{G_{0} \rightarrow B C}^{\left(l_{B C}\right) S S C}= & \sqrt{2 \pi K} \sqrt{\frac{E_{B} E_{C}}{M_{f_{0}}}} M_{G_{0} \rightarrow B C}^{C} \int d \vec{q}_{1} d \vec{q}_{2} \prod_{i=1, . .4} d \vec{p}_{i} \delta\left(\vec{q}_{1}-\vec{p}_{1}-\vec{p}_{2}\right) \\
& \delta\left(\vec{q}_{2}-\vec{p}_{4}-\vec{p}_{3}\right) \Psi_{C}^{\dagger}\left(\vec{p}_{4}, \vec{p}_{2}\right) \Psi_{B}^{\dagger}\left(\vec{p}_{1}, \vec{p}_{3}\right)\left(\overrightarrow{\sigma_{(12)}} \cdot \overrightarrow{\epsilon_{1}}\right)\left(\overrightarrow{\sigma_{(43)}} \cdot \overrightarrow{\epsilon_{2}}\right) \Psi_{G_{0}}\left(\vec{q}_{1}, \overrightarrow{q_{2}}\right) .
\end{aligned}
$$

The flavor matrix elements in the $S U(3)$ flavor symmetry limit are given in Table $\mathbb{I}$. The color transition amplitude $M_{G_{0} \rightarrow B C}^{C}$ is given by

$$
\begin{aligned}
M_{G_{0} \rightarrow B C}^{C} & =\frac{1}{4}<\chi_{C}^{(13)} \chi_{C}^{(42)}\left|\sum_{c=1}^{8} \lambda_{(12)}^{c} A_{1}^{c} \sum_{d=1}^{8} \lambda_{(43)}^{d} A_{2}^{d}\right| \Psi_{C}^{(12)}> \\
& =\frac{1}{24 \sqrt{2}} \sum_{b=1}^{8} \operatorname{Tr}\left[\left(\lambda^{b}\right)^{2}\right]=\frac{\sqrt{2}}{3} .
\end{aligned}
$$

The analytic evaluation of Eq. (22) contains the spin matrix element

$$
\begin{gathered}
<\chi_{S_{B}}(13) \chi_{S_{C}}(42)\left|\left(\overrightarrow{\sigma_{(12)}} \cdot \overrightarrow{\epsilon_{1}}\right)\left(\vec{\sigma}_{(43)} \cdot \overrightarrow{\epsilon_{2}}\right)\right|\left[\epsilon_{1}^{\mu} \otimes \epsilon_{2}^{\mu^{\prime}}\right]_{S=0,2}> \\
=6 \sqrt{\left(2 S_{B}+1\right)\left(2 S_{C}+1\right)}(-)^{S_{C}+1}<S_{B} M_{B} S_{C} M_{C} \mid S \mu+\mu^{\prime}>\left\{\begin{array}{ccc}
1 / 2 & 1 / 2 & S_{B} \\
1 / 2 & 1 / 2 & S_{C} \\
1 & 1 & S
\end{array}\right\}
\end{gathered}
$$

and the spatial overlap 


$$
\begin{aligned}
& \int d \vec{q}_{1} d \vec{q}_{2} \prod_{i=1, . .4} d \overrightarrow{p_{i}} \delta\left(\vec{q}_{1}-\vec{p}_{1}-\vec{p}_{2}\right) \delta\left(\vec{q}_{2}-\vec{p}_{4}-\vec{p}_{3}\right) \delta\left(\vec{q}_{1}+\vec{q}_{2}\right) \\
& \cdot \delta\left(\overrightarrow{p_{1}}+\vec{p}_{3}-\vec{K}\right) \delta\left(\overrightarrow{p_{4}}+\vec{p}_{2}+\vec{K}\right) \Psi_{n_{B} l_{B}}^{\dagger}\left(\vec{p}_{4}, \vec{p}_{2}\right) \Psi_{n_{C} l_{C}}^{\dagger}\left(\vec{p}_{1}, \vec{p}_{3}\right) \Psi_{n=0,1 l=2,0}\left(\vec{q}_{1}, \vec{q}_{2}\right) \\
& =\delta_{l_{f} ; 0} \delta_{m_{f} ; 0} \frac{1}{8} \int d \overrightarrow{q_{1}} d \vec{\rho} \Psi_{n_{B} l_{B}}\left(\overrightarrow{q_{1}}-1 / 2(\vec{K}-\vec{\rho}),-\vec{q}_{1}-1 / 2(\vec{K}+\vec{\rho})\right) \\
& \cdot \Psi_{n_{C} l_{C}}(1 / 2(\vec{K}+\vec{\rho}), 1 / 2(\vec{K}-\vec{\rho})) \Psi_{n=0,1 l=2,0}\left(\vec{q}_{1},-\vec{q}_{1}\right)
\end{aligned}
$$

where $l_{f}$ is the relative orbital angular momentum of the final state with projection $m_{f}$. A complete listing of the analytic results for the amplitudes $M_{G_{0} \rightarrow B C}^{(0) S S C}$ is given in Appendix D. For the decay $G_{0} \rightarrow B C\left(l_{f}\right)$ we obtain the dynamical selection rule that the final state partial wave is restricted to $l_{f}=0$. Hence the decay $f_{0}(M) \rightarrow a_{1} \pi$ will proceed entirely by the quarkonia components of $f_{0}(M)$. Moreover, the D-wave decay amplitude $\left(l_{f}=2\right)$ for the two vector meson final state is forbidden in contrast to quarkonia decay.

With the $Q \bar{Q}$ meson size parameters fixed, $M_{G_{0} \rightarrow B C}^{(0) S S C}$ depends on the glueball size parameter $R_{G_{0}}$. In the constituent picture we expect the mass $M_{G_{0}}$ of the digluonium to be $M_{G_{0}} \approx 2 m_{g}$ with a lattice motivated constituent gluon mass of about $m_{g} \approx 750-850 \mathrm{MeV}$. Assuming equal confinement strength for quarks and gluons on the constituent level we get $R_{G_{0}}=$ $3.65 / \sqrt{2.5} \mathrm{GeV}^{-1}$. With this choice the constituent mass for the lightest quark flavors lies in a reasonable mass range of $300-340 \mathrm{MeV}$. 


\section{TWO-BODY DECAYS OF SCALAR-ISOSCALAR STATES AND THREE-STATE MIXING}

In the following we discuss the application of the decay models developed in the previous sections to the phenomenology of the $f_{0}$ decays. Given the detailed experimental decay data

on the $f_{0}(1500)$, our analysis will focus on this state with decay predictions given for the partner states $f_{0}(1370)$ and $f_{0}(1710)$. After a brief overview of the experimental results for $f_{0}(1500)$, we will illustrate the distinct decay patterns resulting from the unmixed $Q \bar{Q}$ and $G_{0}$ components. In a first analysis we apply the decay models to the three-state mixing schemes constructed in Refs. [4,5]. Then we directly extract the mixing coefficients from a fit to the experimental two-body decay data.

\section{A. Observed decay properties of $f_{0}(1500)$}

The scalar-isoscalar resonance $f_{0}(1500)$ is now clearly established in proton-antiproton $(p \bar{p})$ annihilation reactions by the Crystal Barrel collaboration at CERN [8]. Mass and width of $f_{0}(1500)$ are determined as [8]:

$$
M_{f_{0}(1500)}=1505 \pm 9 \mathrm{MeV} \quad \Gamma_{f_{0}(1500)}=111 \pm 12 \mathrm{MeV} .
$$

For the two-body decay modes $f_{0}(1500) \rightarrow B C$ Crystal Barrel obtains the branching ratios $B R(B C)$ :

$$
\begin{aligned}
B R(\pi \pi) & =29 \pm 7.5 \% \quad B R(\eta \eta)=4.6 \pm 1.3 \% \quad B R\left(\eta \eta^{\prime}\right)=1.2 \pm 0.3 \% \\
B R(K \bar{K}) & =3.5 \pm 0.3 \% \quad B R(4 \pi)=61.7 \pm 9.6 \% .
\end{aligned}
$$

Here, the $4 \pi$ final state includes $\sigma \sigma$ and $\pi^{*}(1300) \pi$ as intermediate decay channels. The allowed $\rho \rho$ decay mode of the $f_{0}(1500)$ is found to be weak [15]. Taking the central value for the width we deduce from the measured branching ratios the partial decay widths $\Gamma(B C)$ :

$$
\begin{aligned}
& \Gamma(2 \pi)=32.2 \pm 8.4 \mathrm{MeV} \quad \Gamma(\eta \eta)=5.2 \pm 1.5 \mathrm{MeV} \quad \Gamma\left(\eta \eta^{\prime}\right)=1.4 \pm 0.4 \mathrm{MeV} \\
& \Gamma(K \bar{K})=3.9 \pm 0.4 \mathrm{MeV} \quad \Gamma(4 \pi)=68.5 \pm 10.7 \mathrm{MeV} .
\end{aligned}
$$


Alternative multi-channel analyses [28] based on data sets from previous experiments have also been performed. The results for the $K \bar{K}$ and $\eta \eta$ modes are within the experimental errors of the Crystal Barrel data. A larger total width $\left(\Gamma_{t o t}=132 \pm 15 \mathrm{MeV}\right)$ and $\pi \pi$ decay width $\left(\Gamma_{\pi \pi}=60 \pm 12 \mathrm{MeV}\right)$ is obtained suggesting a smaller value for $\Gamma(4 \pi)$ than indicated in Eq. (28).

\section{B. Two-body decays of the $Q \bar{Q}$ and $G_{0}$ components}

We first discuss the decay patterns resulting from the individual decay mechanisms of Figs. 2 and 3, coupling to the $Q \bar{Q}$ and the $G_{0}$ components of the $f_{0}$ states, respectively.

In lowest order, neglecting a possible $G_{0} \rightarrow G_{0} G_{0}$ contribution, the decay $f_{0} \rightarrow B C$ will proceed via its quarkonia components $Q \bar{Q}$. In general we consider a mixed quarkonium state

$$
|Q \bar{Q}>=\cos \alpha| n \bar{n}>-\sin \alpha \mid s \bar{s}>.
$$

The dependence of the branching ratios $B R$ for the two-body decays of $f_{0}(1500)$ on the mixing angle $\alpha$ is given in Fig. 因. The results of Fig. 团 correspond to the original discussion in Ref. 四 for the two pseudoscalar meson decay modes of a ${ }^{3} P_{0}$ isoscalar $Q \bar{Q}$ state. But now predictions for all possible two-body decay modes are given.

With the three-state mixing schemes of Ref. 四 $\left(a_{n \bar{n}}=0.43, a_{s \bar{s}}=-0.61\right.$, corresponding to $\left.\alpha=54.8^{\circ}\right)$ and Ref. [5] $\left(a_{n \bar{n}}=0.40, a_{s \bar{s}}=-0.90\right.$, that is $\left.\alpha=66^{\circ}\right)$ we obtain following ratios for the pseudoscalar decay modes

$$
\begin{array}{rr}
B R(\pi \pi): B R(K \bar{K}): B R(\eta \eta): B R\left(\eta \eta^{\prime}\right) \\
=1: 0.21: 0.005: 0.93 & \text { Ref. [4] } \\
=1: 0.97: 0.12: 1.80 & \text { Ref. [5] }
\end{array}
$$

to be compared with the Crystal Barrel results [8] of

$$
\begin{aligned}
& B R(\pi \pi): B R(K \bar{K}): B R(\eta \eta): B R\left(\eta \eta^{\prime}\right) \\
= & 1: 0.12 \pm 0.03: 0.16 \pm 0.06: 0.04 \pm 0.02 .
\end{aligned}
$$


Best agreement with data is achieved for $f_{0}(1500) \approx \mid n \bar{n}>$, for which we obtain

$$
B R(\pi \pi): B R(K \bar{K}): B R(\eta \eta): B R\left(\eta \eta^{\prime}\right)=1: 0.19: 0.16: 0.10
$$

Hence, experimental data for the ratios of two pseudoscalar decay modes of the $f_{0}(1500)$ are consistent with a dominant $n \bar{n}$ interpretation. However, the predicted total width for a pure ${ }^{3} P_{0} n \bar{n}$ state is in conflict with experimental observation. In Fig. 5 we indicate the calculated total width for a $f_{0}(1500)$ state with the $Q \bar{Q}$ configuration of Eq. (29). A pure $n \bar{n}$ state results in $\Gamma_{t o t} \approx 500 \mathrm{MeV}$, which is considerably higher than the observed width. A sizable reduction of the $Q \bar{Q}$ amplitude in the $f_{0}(1500)$, as affected by the admixture of a $G_{0}$ component, leads to a reduction of the total width. For example, the mixing schemes of Refs. [4] and [5] lead to a total width of $\Gamma=111 \mathrm{MeV}$ and $\Gamma=127 \mathrm{MeV}$, respectively. Working in the lowest order of the decay mechanism, i.e. $f_{0}(1500)$ decays by its $Q \bar{Q}$ components, the observed two pseudoscalar decay modes are consistent with a dominant $n \bar{n}$ content, slightly favoring the mixing scheme of Ref. [4]; the possible presence of an admixed $G_{0}$ component is then only reflected in the corresponding reduction of the total width.

When going beyond the two pseudoscalar meson decay modes in the leading order decay scheme, strong deviations from the observed decay pattern of the $f_{0}(1500)$ arise [13]. For the two-body decay modes leading to the $4 \pi$ decay channel, including also charged pion combinations, we obtain

$$
B R(\pi \pi): B R(\rho \rho): B R\left(\pi^{*} \pi\right): B R(\sigma \sigma): B R\left(a_{1} \pi\right)=1: 0.97: 0.14: 0.31: 1.8
$$

Predictions are independent of the mixing angle $\alpha$, since the final states of Eq. (34) only couple to the $n \bar{n}$ configuration. Relative to the $\pi \pi$ channel the $a_{1} \pi$ mode is the strongest decay channel. The predicted large $\rho \rho$ branching ratio with $B R(\rho \rho) \approx B R(\pi \pi)$ is in conflict with the preliminary analysis [15], where the $\rho \rho$ channel is found to be strongly suppressed. Furthermore, for the combined $\sigma \sigma$ and $\pi^{*} \pi$ decay channels with $B R\left(\pi^{*} \rightarrow \sigma \pi\right)=0.1$ [20] we deduce the ratio $B R(4 \pi) / B R(2 \pi)=0.32$, which is much lower than the experimental result of $B R(4 \pi) / B R(2 \pi)=2.1 \pm 0.6$ [8]. The observed suppression of the $\rho \rho$ and enhancement 
of the $4 \pi^{0}$ decay modes are both in strong conflict with a naïve $Q \bar{Q}$ interpretation of the $f_{0}(1500)$ and give further indication for a sizable direct coupling of the gluonic component to the decay channel.

Next we consider the decay pattern evolving from the direct decay of the $G_{0}$ component as indicated in Fig. 35. Branching ratios for the scalar glueball $\left(G_{0}\right)$ decay in dependence on its mass are shown in Fig. 6. A main signature is the strong suppression of the two vector meson decay channels, particularly the $\rho \rho$, over the entire mass range, due to the dynamically forbidden D-wave amplitude. The $\pi^{*} \pi$ and $\sigma \sigma$ decay modes exceed the contribution of the $\pi \pi$ decay channel for a $G_{0}$ mass of about $1500 \mathrm{MeV}$. These results are in clear contrast to those obtained for the $Q \bar{Q}$ decay mechanism. Both, the suppression of the $\rho \rho$ decay mode and the enhancement of the $4 \pi^{0}$ decay channels resulting from the decay of the gluonic component $G_{0}$ are features observed in the $f_{0}(1500)$ decay pattern as discussed previously. For the two pseudoscalar meson decays the results differ from the naïve expectation [4]

$$
B R(\pi \pi): B R(K \bar{K}): B R(\eta \eta): B R\left(\eta \eta^{\prime}\right)=1: \frac{4}{3}: \frac{1}{3}: 0
$$

deduced from flavor symmetric couplings. For a glueball mass of $M_{G_{0}}=1500(1700) \mathrm{MeV}$ we obtain

$$
B R(\pi \pi): B R(K \bar{K}): B R(\eta \eta)=1: 3.7(4): 3.9(4.3)
$$

with $B R\left(\eta \eta^{\prime}\right)=0$ in the $\mathrm{SU}(3)$ flavor symmetry limit. The enhancement of the $K \bar{K} / \pi \pi$ decay ratio relative to the naïve $\mathrm{SU}(3)$ flavor estimate of Eq. (35) is a feature confirmed by recent lattice results [29]. For the given choice of radial meson parameter $\left(R_{K}=2.82 \mathrm{GeV}^{-1}, R_{\eta}=2.48 \mathrm{GeV}^{-1}\right)$, as extracted from tensor meson decay, we obtain $B R(K \bar{K}) \approx B R(\eta \eta)$. Choosing the $\mathrm{SU}(3)$ flavor limit of $R_{K}=R_{\eta}$ leads to $B R(K \bar{K})=4.2 B R(\eta \eta)$, which is again consistent with the analysis of Ref. 29. Hence, the proposed modelling of the $G_{0}$ decay mechanism contains the main features obtained from lattice calculations.

As a measure of flavor symmetry breaking we introduce the parameter $S$ defined by 
$S \equiv<u \bar{u}|V| g>/<s \bar{s}|V| g>\neq 1$ leading to $B R\left(\eta \eta^{\prime}\right) \neq 0$. The flavor matrix elements for $K \bar{K}, \eta \eta, \eta \eta^{\prime}$ decays listed in Table \ correspond to the exact flavor symmetry limit $(S=1)$ and have to be generalised for pure glueball decays $\left(a_{G_{0}}=1\right)$

$$
\begin{aligned}
& M_{G_{0} \rightarrow K \bar{K}}^{F}=2 \sqrt{2} S \\
& M_{G_{0} \rightarrow \eta \eta}^{F}=\sqrt{2}\left(\alpha_{P S}^{2}+S^{2} \beta_{P S}^{2}\right) \\
& M_{G_{0} \rightarrow \eta \eta^{\prime}}^{F}=2 \alpha_{P S} \beta_{P S}\left(1-S^{2}\right) .
\end{aligned}
$$

If we adopt the extreme value of $S^{2}=0.5$ [30] the strength of the $K \bar{K}$ and $\eta \eta$ decay modes will be suppressed by $50 \%$ and $34 \%$, respectively, as compared to the unbroken flavor symmetry couplings.

For scalar glueball masses at $M_{G_{0}}=1300,1500,1700 \mathrm{MeV}$ the reduced available phase space suppresses the $\eta \eta^{\prime}$ mode:

$\begin{array}{ccccc}M_{G_{0}}[\mathrm{MeV}] & B R(\pi \pi): & B R(K \bar{K}): & B R(\eta \eta): & B R\left(\eta \eta^{\prime}\right) \\ 1300 & 1: & 1.3: & 2: & 0.0 \\ 1500 & 1: & 1.8: & 2.6: & 0.08 \\ 1700 & 1: & 2: & 2.9: & 0.28 .\end{array}$

Strong violation of flavor symmetry reduces the $K \bar{K} / \pi \pi$ ratio, while leading to a finite $\eta \eta^{\prime}$ contribution. However, data on charmonium decay involving hard gluons indicate no significant symmetry breaking [4]. We therefore choose to set $\mathrm{S}=1$ in the following discussion. Decay data on $f_{0}(1500)$ possess qualitative features arising both from the decay of a $Q \bar{Q}$

(here dominantly $n \bar{n}$ ) and a $G_{0}$ configuration, where a pure $Q \bar{Q}$ or $G_{0}$ state interpretation of the $f_{0}(1500)$ is excluded. Given this simple first analysis of the decay pattern of the $f_{0}(1500)$ we are naturally led to a mixing scheme where both components are present.

\section{Decay analysis in the three-state mixing schemes}

In the following we give a decay analysis for the $f_{0}$ states defined by the three-state mixing schemes of Refs. [四 and [5]. Now, the couplings both of the $Q \bar{Q}$ and $G_{0}$ components 
to the two-meson decay channels are included.

When the physical $f_{0}$ mesons are given as eigenstates of the interaction Hamiltonian $H_{I}$ of Eq. (1), the partial decay widths of the process $f_{0} \rightarrow B C$ are defined by Eq. (5) with the ratio of coupling strengths $\kappa$ and the phase angle $\phi$ left as free parameters. The structure of $H_{I}$ implies unbroken flavor symmetry, hence we choose $S=1$. For the $f_{0}(1500)$ decays we give in Table III the squared decay amplitudes and the interference terms as defined in Eq. (5), where mass averaging as given by Eq. (6) is already included. The mass distribution of $f_{0}(1500)$ is parameterised with the values of Eq. (26).

The dependence of the partial decay widths $\Gamma(B C)$ on the mixing coefficients $a_{i}(i=$ $\left.n \bar{n}, s \bar{s}, G_{0}\right)$ in the schemes of Ref. [4] (三 scheme A) and Ref. [5] (三 scheme B) are studied by adjusting $\kappa$ and $\phi$ to the Crystal Barrel data for $f_{0}(1500)$ decays. First we have determined $\kappa$ and $\cos \phi$ from a fit to the observed decay modes $f_{0}(1500) \rightarrow K \bar{K}$ and $\pi \pi$. For both mixing schemes we obtain parameter values resulting in a $f_{0} \rightarrow \eta \eta$ decay width in good agreement with the experimental number. This implies phenomenologically, that a $G_{0} \rightarrow G_{0} G_{0}$ contribution to the $\eta \eta$ decay channel can be neglected. For this reason, we also take the $\eta \eta$ decay mode into account when determining $\cos \phi$ and $\kappa$ from a more restrictive fit. The partial decay widths have a rather weak dependence on the parameterisation of the mass distribution of the physical $f_{0}$ states. Therefore, the discussion [8] on the detailed relationship between the $f_{0}(1370)$ and $f_{0}(400-1200)$ states is peripheral to our analysis. For our numerical results we use the resonance parameters $M_{f_{0}(1370)}=1360 \mathrm{MeV}, \Gamma_{f_{0}(1370)}=350 \mathrm{MeV}$ [8], and $M_{f_{0}(1710)}=1697 \mathrm{MeV}, \Gamma_{f_{0}(1710)}=175 \mathrm{MeV}$ [10].

The mixing coefficients in scheme A [4] are given as

$$
\left(\begin{array}{l}
\mid f_{0}(1370)> \\
\mid f_{0}(1500)> \\
\mid f_{0}(1710)>
\end{array}\right)=\left(\begin{array}{ccc}
0.86 & 0.13 & -0.5 \\
0.43 & -0.61 & 0.61 \\
0.22 & 0.76 & 0.6
\end{array}\right)\left(\begin{array}{l}
\mid n \bar{n}> \\
\mid s \bar{s}> \\
\mid G_{0}>
\end{array}\right)
$$

From a fit to the $f_{0}(1500) \rightarrow \pi \pi, K \bar{K}, \eta \eta$ decay widths we deduce $\cos \phi=-0.67$ and $\kappa=0.095 \mathrm{GeV}^{-2}$. Predictions for the partial decay widths of the physical $f_{0}$ states are 
presented in Table $\mathbb{I V}$. For $f_{0}(1500)$ the predicted $\eta \eta^{\prime}$ decay width is considerably lower than the Crystal Barrel value, but the obtained ratio $\Gamma\left(\eta \eta^{\prime}\right) / \Gamma(\eta \eta)$ agrees with the GAMS value of $2.7 \pm 0.8$ for $f_{0}(1590)$ [10]. It is generally believed that $f_{0}(1500)$ and $f_{0}(1590)$ are manifestations of a single state, but then the large deviation in the experimental data for the $\eta \eta^{\prime}$ decay mode has to be clarified. Compared to the lowest order decay analysis, where only the coupling to the $Q \bar{Q}$ component is retained, the $\rho \rho$ mode is more suppressed and the $4 \pi$ channel enhanced. We obtain $B R(\pi \pi): B R(\rho \rho): B R(4 \pi)=1: 0.65: 0.46-0.76$ for $B R\left(\pi^{*} \rightarrow \sigma \pi\right)=0.1-1$, still in deviation from the Crystal Barrel data.

In the mixing scheme B [5] the amplitudes for the physical states are given as

$$
\left(\begin{array}{l}
\mid f_{0}(1370)> \\
\mid f_{0}(1500)> \\
\mid f_{0}(1710)>
\end{array}\right)=\left(\begin{array}{ccc}
0.84 & 0.28 & -0.46 \\
0.40 & -0.9 & 0.19 \\
0.36 & 0.34 & 0.87
\end{array}\right)\left(\begin{array}{l}
\mid n \bar{n}> \\
\mid s \bar{s}> \\
\mid G_{0}>
\end{array}\right)
$$

From an analogous fit we obtain $\cos \phi=-0.953$ and $\kappa=0.437 \mathrm{GeV}^{-2}$. The predicted twobody partial decay widths are given in Table $\mathrm{V}$. Compared to the mixing scheme $\mathrm{A}, f_{0}(1500)$ has a reduced $G_{0}$ component, but the experimental data demand a significant $G_{0} \rightarrow B C$ contribution in fitting the $\pi \pi, K \bar{K}$ and $\eta \eta$ decays. This is balanced by giving the $G_{0} \rightarrow B C$ transition a stronger weight than in the mixing scheme A, resulting in a higher value for $\kappa$. Again, the predicted strength of the $\eta \eta^{\prime}$ decay mode overestimates the experimental value, while similar results are obtained for the $\rho \rho$ and $4 \pi$ decay modes. For mixing scheme B we obtain $B R(\pi \pi): B R(\rho \rho): B R(4 \pi)=1: 0.46: 0.55-0.97$ for $B R\left(\pi^{*} \rightarrow \sigma \pi\right)=0.1-1$. In both schemes $a_{1} \pi$ is a dominant decay mode with $B R(\pi \pi) \approx B R\left(a_{1} \pi\right)$. With our fitting procedure, that is adjusting the relative strength of the $G_{0}$ decay contribution to the $\pi \pi, K \bar{K}$ and $\eta \eta$ decay channels of the $f_{0}(1500)$, similar results are obtained for the decay pattern of the $f_{0}(1500)$ in both mixing schemes. Particular predictions for the decay modes of the partner states $f_{0}(1370)$ and $f_{0}(1710)$ can be dramatically different in the two mixing schemes. In scheme A the total width for $f_{0}(1710)$ is in good agreement with the experimental result of $\Gamma=175 \pm 9 \mathrm{MeV}(\Gamma=133 \pm 14 \mathrm{MeV})$ [10], but scheme B predicts a unobservable decay 
width of $\Gamma \approx 2000 \mathrm{MeV}$. Furthermore, for $f_{0}(1710)$ we get

$$
B R(\pi \pi) / B(K \bar{K}))=\left\{\begin{array}{l}
0.93 \text { for scheme A } \\
0.60 \text { for scheme B }
\end{array}\right.
$$

to be compared with the experimental value of $0.39 \pm 0.14$ [10].

The two mixing schemes predict rather similar results for the $f_{0}(1370)$ state. In both schemes $f_{0}(1370)$ has dominant $\rho \rho$ and $a_{1} \pi$ decay modes. The ratio $B R(K \bar{K}) /(B R(\eta \eta)$ is found to be

$$
B R(K \bar{K}) / B R(\eta \eta)=\left\{\begin{array}{c}
2.6 \text { for scheme A } \\
1.45 \text { for scheme B }
\end{array}\right.
$$

to be compared with the Crystal Barrel value of $B R(K \bar{K}) / B R(\eta \eta) \approx 2.5[8]$.

The main conclusions of this section remain unchanged if we use for $f_{0}(1500)$ a larger $\pi \pi$ decay width as reported in Ref. [28], since a corresponding fit leads to similar values for $\kappa$ and $\cos \phi$ as for the adjustment to the Crystal Barrel data.

Inclusion of the direct coupling of the $G_{0}$ component of the mixed $f_{0}(1500)$ state to the twomeson decay channels gives an improved description of its decay features. For both proposed mixing schemes predictions for the $f_{0}(1500)$ decays are very similar, since the difference in size of the $G_{0}$ amplitude is compensated by adjusting the strength of the $G_{0} \rightarrow(Q \bar{Q})(Q \bar{Q})$ transition.

Also, predictions for the $f_{0}(1370)$ state do not differ too much, since both schemes assign a dominant $n \bar{n}$ component to this state. The key difference rests on predictions for the partner state $f_{0}(1710)$. We have shown that a reduced $G_{0}$ component in $f_{0}(1500)$, as proposed by Ref. [5], is incompatible with the existence of a $f_{0}$ state at $1700 \mathrm{MeV}$ as a narrow resonance. Conversely, if the existence of a narrow scalar component of the $f_{j=0,2}(1710)$ [10] state is confirmed, then a non-negligible glueball component is residing in the $f_{0}(1500)$ state. 


\section{Mixing amplitudes}

Given the shortcomings of the proposed mixing schemes of Refs. [4,5] we now directly extract the mixing amplitudes of the $f_{0}$ states in the given decay formalism. We proceed in an analogous fashion, requiring a fit to the experimental data on $f_{0}(1500)$ decays, while demanding the existence of a narrow resonance $f_{0}(1710)$ as an additional requirement.

First, we start with the unspecified mixing amplitudes of the $f_{0}(1500)$ state vector as defined by Eq. (2). The mixing amplitudes of $f_{0}(1500)$ are determined from a fit to the experimental data of the $\pi \pi, K \bar{K}$ and $\eta \eta$ decay modes with the restriction of a weak $\rho \rho$ decay channel. Exact flavor symmetry $(S=1)$ for the quark-gluon coupling is assumed. Good agreement with the experimental data requires $a_{n \bar{n}} \cdot a_{s \bar{s}}<0$, i.e. a destructive interference between the $n \bar{n}$ and $s \bar{s}$ flavors, and we get

$$
\left|f_{0}(1500)>=0.314\right| n \bar{n}>-0.581|s \bar{s}>+0.751| G_{0}>
$$

with the ratio of coupling strengths $\kappa=0.1 \mathrm{GeV}^{-2}$ and the phase factor $\cos \phi=-0.92$. The fit to the $f_{0}(1500)$ decays alone does not fix the phase of the $a_{G_{0}}$ amplitude relative to the quarkonia coefficients. Instead we obtain the condition that $a_{G_{0}} \cdot \cos \phi<0$. For the $f_{0}(1500)$ state vector we have taken $a_{G_{0}}>0$ and $\cos \phi<0$ for reasons given later. The relative phases between the state amplitudes of Eq. (43) are the same as in mixing schemes A and B. For the full set of partial decay widths we obtain the results given in Table $\mathrm{VI}$. Compared to mixing scheme A the $\rho \rho$ mode of $f_{0}(1500)$ is suppressed by a factor of two. This is achieved by enhancing the $G_{0}$ amplitude since the $\rho \rho$ suppression for $f_{0}(1500)$ is driven by the gluonic component (see Sec. IV). An alternative fit to obtain $\rho \rho$ suppression requires a strong $s \bar{s}$ component and a larger value for $\kappa$ as resulting for example from the mixing scheme B. However, the existence of a narrow $f_{0}(1710)$ state rules out this possibility.

For the $4 \pi / 2 \pi$ decay branching ratio we obtain $B R(4 \pi) / B R(2 \pi)=0.57-1.04$ using $B R\left(\pi^{*} \rightarrow \sigma \pi\right)=0.1-1$, where recent results [20] for $\pi^{*} \rightarrow \sigma \pi$ favor the lower value. This is still in deviation from the Crystal Barrel value of $B R(4 \pi) / B R(2 \pi)=2.1 \pm 0.6$ [8]. 
The disagreement possibly hints at a sizable $G_{0}$ component in $\sigma$. A strong coupling of $\sigma$ to $G_{0}$ is supported by the experimental finding [10] that $\sigma \sigma$ is the largest hadronic branching ratio for the charmonium states $\chi_{0}$ and $\chi_{2}$. In this case, the lowest order decay mechanism $G_{0} \rightarrow G_{0} G_{0}$, neglected here, can enhance the $\sigma \sigma$ and therefore the $4 \pi$ decay mode. The predicted ratio $B R\left(\eta \eta^{\prime}\right) / B R(\eta \eta)=2.2$ is in agreement with the GAMS value of $2.7 \pm 0.8$ [10], but in conflict with the Crystal Barrel result of $0.27 \pm 0.10$ [8]. Breaking of flavor symmetry at the quark-gluon vertex, that is $S \neq 1$, will further enhance the $\eta \eta^{\prime}$ decay width.

With mass and eigenstate of $f_{0}(1500)$ fixed at $1500 \mathrm{MeV}$ and by Eq. (43), respectively, we deduce for the bare masses $m_{i}\left(i=n \bar{n}, s \bar{s}, G_{0}\right)$ entering in $H_{I}$ of Eq. (1)

$$
\begin{gathered}
m_{n \bar{n}}=1500 \mathrm{MeV}-3.38 z, \quad m_{s \bar{s}}=1500 \mathrm{MeV}+1.29 z, \\
m_{G_{0}}=1500 \mathrm{MeV}+0.18 z
\end{gathered}
$$

with the mixing strength $z>0$. For the bare masses we obtain the level ordering $m_{n \bar{n}}<$ $m_{G_{0}}<m_{s \bar{s}}$, which is independent of $\mathrm{z}$ and corresponds to that proposed originally in Ref. [4]. When taking the alternative sign pattern of $a_{G_{0}}<0$ and $\cos \phi>0$ for the fit to the $f_{0}(1500)$ decays, we obtain the unphysical level ordering of the bare states with $m_{n \bar{n}}>m_{s \bar{s}}>m_{G_{0}}$, we hence exclude this choice. Additionally, for the masses $M_{<(>)}$of the mixing partners lying above $\left(M_{>}\right)$and below $\left(M_{<}\right) f_{0}(1500)$ we obtain:

$$
\begin{aligned}
& M_{>}=1500 \mathrm{MeV}+2 z \\
& M_{<}=1500 \mathrm{MeV}-3.9 z .
\end{aligned}
$$

For consistency, we call these states $f_{0}(1370)$ and $f_{0}(1710)$ and use the values for mass and total width given in the previous section for the parameterisation of the respective mass distributions. For the mixing partners of the $f_{0}(1500)$ we obtain the glueball-quarkonia content

$$
\left|f_{0}(1710)>=0.149\right| n \bar{n}>+0.811|s \bar{s}>+0.565| G_{0}>
$$

and 


$$
\left|f_{0}(1370)>=0.938\right| n \bar{n}>\quad+0.070|s \bar{s}>\quad-0.341| G_{0}>,
$$

where the amplitudes are independent of the particular value of z. Predictions for the corresponding partial decay widths of $f_{0}(1710)$ and $f_{0}(1370)$ are also given in Table $\left.\nabla\right]$. The total width and the ratio $B R(\pi \pi) / B R(K \bar{K})=0.57$ of the $f_{0}(1710)$ are in good agreement with the experimental results of of $\Gamma=175 \pm 9 \mathrm{MeV}(133 \pm 14 \mathrm{MeV})$ [10] and $B R(\pi \pi) / B R(K \bar{K})=0.39 \pm 0.14$ for $f_{j=0,2}(1710)$ [10]. For the $f_{0}(1370)$ the predicted ratio of $B R(K \bar{K}) / B R(\eta \eta)=2.7$ is to be compared to the experimental value $\approx 2.5$ [ 8 . For the total width we get $\Gamma_{t o t}=479 \mathrm{MeV}$, somewhat larger than the Crystal Barrel value of $\Gamma=351 \pm 41 \mathrm{MeV}[8]$.

For a mixing energy $z=43 \pm 31 \mathrm{MeV}$ [14], as deduced from lattice QCD in the quenched approximation, we obtain $m_{G_{0}}=1508 \pm 6 \mathrm{MeV}$, in agreement with the lattice result of $m_{G_{0}}=1.65 \pm 0.15 \mathrm{GeV}$ [3]. For the bare masses we get $m_{n \bar{n}}=1355 \pm 105 \mathrm{MeV}$ and $M_{s \bar{s}}=1556 \pm 40 \mathrm{MeV}$. The values for the glueball-quarkonia mixing matrix element $\mathrm{z}$ favored by previous approaches are $z=77 \mathrm{MeV}$ [5], $z=64 \pm 13 \mathrm{MeV}$ [14] and $z \approx 100 \mathrm{MeV}$, where later value is deduced from the full nonperturbative three-state mixing scheme of Ref. [4]. Hence, semiphenomenological extractions of $\mathrm{z}$ favor a value near the upper limit set by the lattice result.

Choosing an intermediate value of $z \approx 80 \mathrm{MeV}$ we obtain for the physical masses of the mixing partners given by Eq. (45), $M_{>}=1660 \mathrm{MeV}$ and $M_{<}=1190 \mathrm{MeV}$, where $M_{<}$ is lower than the mass of the $f_{0}(1370)$. A similar result is obtained from the full mixing approach of Ref. [ [ब], which corresponds qualitatively to our extracted scheme.

Given our fit to the $f_{0}(1500)$ decays, we obtain a physical mass $M_{<}$and a total width which deviate from the corresponding data on $f_{0}(1370)$. Therefore, within the framework of a three-state mixing scheme, the lower lying partner of $f_{0}(1500)$ could be identified with the very broad structure around $1100 \mathrm{MeV}$ [4], called $f_{0}(400-1200)$ [10]. Alternatively, the Crystal Barrel state $f_{0}(1370)$ could be the high mass tail of $f_{0}(400-1200)$. However, given the ansatz of Eq. (1), the most stable and testable consequences of our mixing scheme con- 
cern predictions for all the two-body decay modes of the $f_{0}$ states as given in Table $V 1$. Given the sensitivity of the decay pattern on the particular size of the $G_{0}$ and $Q \bar{Q}$ components in the $f_{0}$ states only, a full experimental determination provides a stringent test. 


\section{SUMMARY AND CONCLUSIONS}

We have performed a detailed study of the two-body decay properties of the scalarisoscalar $f_{0}(1370), f_{0}(1500)$ and $f_{0}(1710)$ states resulting from the mixture of the lowest lying scalar glueball with the isoscalar quarkonia states of the $0^{++}$nonet. In the decay analysis we have taken into account the coupling of the quarkonia and glueball components of the $f_{0}$ states to the quarkonia components of the two-meson final state, where the decay dynamics is guided by strong coupling QCD. Leading order corresponds to the transitions $Q \bar{Q} \rightarrow(Q \bar{Q})(Q \bar{Q})$ and $G_{0} \rightarrow G_{0} G_{0}$, where the latter transition, which can contribute to $\eta \eta, \eta \eta^{\prime}$ and $\sigma \sigma$ final states, is omitted due to its ill-constrained nature. The coupling of the quarkonia component $(Q \bar{Q})$ of the $f_{0}$ states is described in the framework of the ${ }^{3} P_{0}$ pair creation model, which is successful in the phenomenology of OZI-allowed meson decay. In next-to-leading order we obtain a direct coupling of the $G_{0}$ component of the $f_{0}$ states to the quarkonia component of the decay channel. The corresponding transition $G_{0} \rightarrow(Q \bar{Q})(Q \bar{Q})$ is modelled by resorting to a scalar digluonium wavefunction for $G_{0}$ as given by cavity QCD. Predictions for the two-pseudoscalar decay channels, that is $\pi \pi, K \bar{K}$ and $\eta \eta$, are in rough agreement with recent lattice results in the limit of unbroken flavor SU(3).

Our analysis on the two-body decay modes of the $f_{0}$ states focuses on the $f_{0}(1500)$, where detailed experimental data are available. In comparison to the observed decay properties of $f_{0}(1500)$ we elaborate the distinct decay patterns resulting from the unmixed $Q \bar{Q}$ and $G_{0}$ components. Taking into account the coupling of the $Q \bar{Q}$ components of the $f_{0}(1500)$ only, which corresponds to the leading order decay mechanism of Ref. [4], data on twopseudoscalar decay channels are consistent with a dominant $n \bar{n}$ structure residing in this state. However, $f_{0}(1500)$ is too narrow for a simple $n \bar{n}$ structure, implying a sizable reduction of its quarkonia components as resulting from glueball-quarkonia mixing. When going beyond the two-pseudoscalar decay channels large deviations from the observed decay pattern of the $f_{0}(1500)$ arise. The large $4 \pi$ branching ratio, fed by the $\sigma \sigma$ and $\pi^{*} \pi$ decay channels, and the suppression of $\rho \rho$ is in clear conflict with the simple leading order 
analysis of $f_{0}(1500)$, where coupling to its $Q \bar{Q}$ component is taken, and points towards the relevance of a possible glueball component. Coupling of the $G_{0}$ component of the $f_{0}(1500)$ to the quarkonia component of the two-body final state yields a significantly different decay pattern. In the two-pseudoscalar sector strong $K \bar{K}$ and $\eta \eta$ decay modes are found. Furthermore, $\pi^{*} \pi$ and $\sigma \sigma$ dominate over the $\pi \pi$ decay channel, whereas $\rho \rho$ is strongly suppressed. This latter feature of a strongly reduced $\rho \rho$ coupling to $G_{0}$ is also obtained in a recent work [31], where the coupling of $G_{0}$ to the pionic decay channel is set up in an effective linear sigma model. Both observed features of the $f_{0}(1500)$, the enhancement of the $4 \pi^{0}$ decay channels and the reduction of the $\rho \rho$ channel, are driven by its gluonic component.

In a next step we have investigated the decay properties of the $f_{0}(1370), f_{0}(1500)$ and $f_{0}(1710)$ states in the proposed three-state mixing schemes of Refs. [4,5]. Due to the inclusion of the $G_{0} \rightarrow(Q \bar{Q})(Q \bar{Q})$ mechanism in the decay analysis we were able to discriminate between the mixing schemes, in particular with respect to the orthogonal physical consequences for the $f_{0}(1710)$ state. A negligible $G_{0}$ component in $f_{0}(1500)$ as proposed by Ref. [5] is incompatible with the existence of a narrow $f_{0}$ state in the $1700 \mathrm{MeV}$ mass region; hence our first analysis of the two-body decays favors the mixing scheme of Ref. [4].

Finally, we have extracted a three-state mixing scheme based on a detailed fit to the experimental $f_{0}(1500)$ decays, while demanding the existence of a narrow $f_{0}$ mixing partner in the $1700 \mathrm{MeV}$ mass region. For the bare masses before mixing we obtain the level ordering $m_{n \bar{n}}<m_{s \bar{s}}<m_{G_{0}}$, corresponding qualitatively to the scheme originally proposed in Ref. [4]. Adjustment of the gluonic decay mechanism leads to a proper description of the observed two-pseudoscalar decay modes of $f_{0}(1500)$ with the exception of $\eta \eta^{\prime}$. Accordingly, we obtain a significant $\rho \rho$ suppression coupled at the same time to the enhancement of the $4 \pi^{0} / 2 \pi$ ratio, in line with experimental requirements. A full reduction of the observed $4 \pi / 2 \pi$ ratio requires a strong $\sigma \sigma$ mode. Both deviations in the $\eta \eta^{\prime}$ and $\sigma \sigma$ decay channels suggest a non-negligible leading order $G_{0} \rightarrow G_{0} G_{0}$ contribution, which was neglected here. A key signature for the extracted state composition of $f_{0}(1500)$ is the prediction for the $a_{1} \pi$ decay mode with $B R\left(a_{1} \pi\right) \approx B R(\pi \pi)$. A pure $Q \bar{Q}$ configuration or equivalently, application of the 
leading order decay mechanism to the mixed $f_{0}(1500)$ state results in $B R\left(a_{1} \pi\right) \approx 2 B R(\pi \pi)$. Hence an experimental determination of the $a_{1} \pi$ decay channel of $f_{0}(1500)$ is desirable to further quantify the relevance of its gluonic component.

The resulting predictions for the decays of the $f_{0}(1370)$ and $f_{0}(1710)$ states are consistent with the limited experimental data [8,10] on pseudoscalar decay modes. Clearly a search for

the decay channels feeding the $4 \pi$ decay products is most relevant in clarifying the nature of these partner states. Predictions for the total widths of $f_{0}(1370)$ and $f_{0}(1710)$ roughly agree with the observed values [8,10]; the physical mass spectrum resulting from the extracted mixing scheme points towards a sizable influence of the broad $f_{0}$ structure at $1100 \mathrm{MeV}$ [8], which should be clarified.

The quantitative predictions of our analysis emphasise the role of the gluonic components in the $f_{0}$ states and its observable consequences in the two-body decay modes. Hence a full determination of the hadronic decay properties provides a sensitive test on the nature of the $f_{0}$ states, revealing the intrusion of the glueball ground state in the scalar-isoscalar meson spectrum.

\section{ACKNOWLEDGMENTS}

This work was supported in part by the Graduiertenkolleg "Struktur und Wechselwirkung von Hadronen und Kernen" (DFG Mu705/3) and by a grant of the Deutsches Bundesministerium für Bildung und Forschung (contract No. 06 Tü 887). 


\section{APPENDIX A: SPATIAL WAVE FUNCTIONS}

For the spatial part of the wave functions used in the text we use harmonic oscillators $\Psi_{n l}\left(\vec{p}_{i}, \vec{p}_{j}\right)$ which are defined in the limit of equal constituent masses as

$$
\begin{aligned}
\Psi_{n l}\left(\overrightarrow{p_{i}}, \overrightarrow{p_{j}}\right)= & N_{n l}\left(\frac{R}{2}\right)^{l}\left|\overrightarrow{p_{i}}-\vec{p}_{j}\right|^{l} \exp \left\{-\frac{1}{8} R^{2}\left(\vec{p}_{i}-\vec{p}_{j}\right)^{2}\right\} \\
& L_{n}^{l+1 / 2}\left(\frac{R^{2}}{4}\left(\overrightarrow{p_{i}}-\vec{p}_{j}\right)^{2}\right) Y_{l m}\left(\vec{p}_{i}-\hat{p_{j}}\right)
\end{aligned}
$$

with the internal momentum $p_{i(j)}$ and the size parameter $R$. The normalisation constant $N_{n l}$ depends on the radial and orbital quantum numbers n, l with

$$
N_{n l}=\left[\frac{2(n !) R^{3}}{\Gamma(n+l+3 / 2)}\right]^{1 / 2} .
$$

The Laguerre polynomials are given by

$$
L_{n}^{l+1 / 2}(p)=\sum_{k=0}^{n} \frac{(-)^{k} \Gamma(n+l+3 / 2)}{k ! \Gamma(k+l+3 / 2)} p^{k} .
$$

For the $Q \bar{Q}$ mesons we use:

$$
(n l)=\left\{\begin{array}{l}
(00) \text { for } \pi, \eta, \eta^{\prime}, K, \bar{K}, \rho, \omega \\
(01) \text { for } a_{1}, \sigma, f_{0} \\
(10) \text { for } \pi^{*}(1300)
\end{array}\right.
$$




\section{APPENDIX B: QUARKONIA DECAY AMPLITUDES}

In the following we give the full expressions for the spin-spatial-color amplitudes $M_{Q \bar{Q} \rightarrow B C}^{\left(l_{B C}\right) S S C}$ defined in Eq. (12) as obtained for the quarkonia decay process $Q \bar{Q} \rightarrow B C$. For equal size parameters $\left(\equiv R_{B}\right)$ of the final state mesons $\mathrm{B}$ and $\mathrm{C}$ we obtain:

1) $Q \bar{Q} \rightarrow \pi \pi, \eta \eta, \eta \eta^{\prime}, K \bar{K}$

$$
\begin{aligned}
M_{Q \bar{Q} \rightarrow B C}^{(0) S S C}= & \sqrt{\frac{E_{B} E_{C}}{M_{f_{0}}}} \frac{8}{\pi^{1 / 4}} \frac{R_{Q \bar{Q}}^{5 / 2} R_{B}^{3}}{\left(R_{Q \bar{Q}}^{2}+2 R_{B}^{2}\right)^{5 / 2}} \exp \left\{-\frac{1}{4}\left(\frac{R_{Q \bar{Q}}^{2} R_{B}^{2}}{R_{Q \bar{Q}}^{2}+2 R_{B}^{2}}\right) K^{2}\right\} \\
& {\left[K^{1 / 2}-\frac{R_{B}^{2}\left(R_{Q \bar{Q}}^{2}+R_{B}^{2}\right)}{3\left(R_{Q \bar{Q}}^{2}+2 R_{B}^{2}\right)} K^{5 / 2}\right] }
\end{aligned}
$$

2) $Q \bar{Q} \rightarrow \rho \rho, \omega \omega$

$$
\begin{aligned}
M_{Q \bar{Q} \rightarrow B C}^{\left(l_{B C}\right) S S C}= & \sqrt{\frac{E_{B} E_{C}}{M_{f_{0}}}} \frac{8}{\sqrt{3} \pi^{1 / 4}} \frac{R_{Q \bar{Q}}^{5 / 2} R_{B}^{3}}{\left(R_{Q \bar{Q}}^{2}+2 R_{B}^{2}\right)^{5 / 2}} \exp \left\{-\frac{1}{4}\left(\frac{R_{Q \bar{Q}}^{2} R_{B}^{2}}{R_{Q \bar{Q}}^{2}+2 R_{B}^{2}}\right) K^{2}\right\} \\
& \cdot \begin{cases}K^{1 / 2}-\frac{R_{B}^{2}\left(R_{Q \bar{Q}}^{2}+R_{B}^{2}\right)}{3\left(R_{Q \bar{Q}}^{2}+2 R_{B}^{2}\right)} K^{5 / 2} \text { for } & l_{B C}=0 \\
-\sqrt{\frac{8}{9}} \cdot \frac{R_{B}^{2}\left(R_{Q \bar{Q}}^{2}+R_{B}^{2}\right)}{R_{Q \bar{Q}}^{2}+R_{B}^{2}} K^{5 / 2} \text { for } \quad l_{B C}=2\end{cases}
\end{aligned}
$$

3) $Q \bar{Q} \rightarrow \sigma \sigma$

$$
\begin{aligned}
M_{Q \bar{Q} \rightarrow \sigma \sigma}^{(0) S S C}= & \sqrt{\frac{E_{\sigma} E_{\sigma}}{M_{f_{0}}}} \frac{4}{9 \pi^{1 / 4}} \frac{R_{Q \bar{Q}}^{5 / 2} R_{B}^{5}}{\left(R_{Q \bar{Q}}^{2}+2 R_{B}^{2}\right)^{7 / 2}} \exp \left\{-\frac{1}{4}\left(\frac{R_{Q \bar{Q}}^{2} R_{B}^{2}}{R_{Q \bar{Q}}^{2}+2 R_{B}^{2}}\right) K^{2}\right\} \\
& {\left[60 K^{1 / 2}+\frac{11 R_{Q \bar{Q}}^{4}+4 R_{B}^{2}\left(R_{Q \bar{Q}}^{2}+R_{B}^{2}\right)}{R_{Q \bar{Q}}^{2}+2 R_{B}^{2}} K^{5 / 2}-\frac{R_{Q \bar{Q}}^{4} R_{B}^{2}\left(R_{Q \bar{Q}}^{2}+R_{B}^{2}\right)}{\left(R_{Q \bar{Q}}^{2}+2 R_{B}^{2}\right)^{2}} K^{9 / 2}\right] }
\end{aligned}
$$

4) $Q \bar{Q} \rightarrow \pi^{*}(1300) \pi$

$$
M_{Q \bar{Q} \rightarrow \pi^{*}(1300) \pi}^{(0) S C}=\sqrt{\frac{E_{\pi} E_{\pi^{*}}}{M_{f_{0}}}} \frac{2^{3 / 2}}{3^{3 / 2} \pi^{1 / 4}} \frac{R_{Q \bar{Q}}^{5 / 2} R_{B}^{3}}{\left(R_{Q \bar{Q}}^{2}+2 R_{B}^{2}\right)^{7 / 2}} \exp \left\{-\frac{1}{4}\left(\frac{R_{Q \bar{Q}}^{2} R_{B}^{2}}{R_{Q \bar{Q}}^{2}+2 R_{B}^{2}}\right) K^{2}\right\}
$$




$$
\begin{aligned}
& {\left[6 \cdot\left(3 R_{Q \bar{Q}}^{2}-4 R_{B}^{2}\right) K^{1 / 2}-\frac{R_{Q \bar{Q}}^{2} R_{B}^{2}\left(13 R_{Q \bar{Q}}^{2}+6 R_{B}^{2}\right)}{R_{Q \bar{Q}}^{2}+2 R_{B}^{2}} K^{5 / 2}\right.} \\
& \left.+\frac{R_{Q \bar{Q}}^{4} R_{B}^{4}\left(R_{Q \bar{Q}}^{2}+R_{B}^{2}\right)}{\left(R_{Q \bar{Q}}^{2}+2 R_{B}^{2}\right)^{2}} K^{9 / 2}\right]
\end{aligned}
$$

5) $Q \bar{Q} \rightarrow a_{1} \pi$

$$
M_{Q \bar{Q} \rightarrow a_{1} \pi}^{(1) S S C}=\sqrt{\frac{E_{a_{1}} E_{\pi}}{M_{f_{0}}}} \frac{2^{4}}{3 \pi^{1 / 4}} \frac{R_{f_{0}}^{5 / 2} R_{B}^{4}}{\left(R_{Q \bar{Q}}^{2}+2 R_{B}^{2}\right)^{5 / 2}} \exp \left\{-\frac{1}{4}\left(\frac{R_{Q \bar{Q}}^{2} R_{B}^{2}}{R_{Q \bar{Q}}^{2}+2 R_{B}^{2}}\right) K^{2}\right\} K^{3 / 2}
$$




\section{APPENDIX C: TRANSFORMATION BRACKETS}

The relevant values for the transformation brackets $<n l, N L, g \mid n_{1} l_{1}, n_{2} l_{2}, g>$ used in Eq. (19) are:

1) $g=0$

$$
\begin{aligned}
& <00,10,0 \mid 01,01,0>=\frac{1}{\sqrt{2}} \\
& <01,01,0 \mid 01,01,0>=0 \\
& <10,00,0 \mid 01,01,0>=-\frac{1}{\sqrt{2}}
\end{aligned}
$$

2) $g=1$

$$
<01,01,1 \mid 01,01,1>=1
$$

3) $g=2$

$$
\begin{aligned}
& <00,02,2 \mid 01,01,2>=\frac{1}{\sqrt{2}} \\
& <01,01,2 \mid 01,01,2>=0 \\
& <02,00,2 \mid 01,01,2>=-\frac{1}{\sqrt{2}}
\end{aligned}
$$




\section{APPENDIX D: DIGLUONIUM DECAY AMPLITUDES}

The spin-spatial-color matrix elements $M_{G_{0} \rightarrow B C}^{S S C}$, defined in Eq. (22), for the decay of the scalar digluonium state $G_{0}$ (size parameter $R_{G_{0}}$ ) into the final state mesons B C (size parameter $R_{B}$ ) are:

1) $G_{0} \rightarrow \pi \pi, \eta \eta, \eta \eta^{\prime}, K \bar{K}(\equiv 2 P S)$

$$
M_{G_{0} \rightarrow 2 P S}^{(0) S S C}=\sqrt{\frac{E_{B} E_{C}}{M_{f_{0}}}} \frac{32}{3} \sqrt{2} \pi^{7 / 4} \frac{R_{G_{0}}^{3 / 2}\left(R_{B}^{2}-2 R_{G_{0}}^{2}\right)}{\left(R_{B}^{2}+2 R_{G_{0}}^{2}\right)^{5 / 2}} K^{1 / 2}
$$

2) $G_{0} \rightarrow \rho \rho, \omega \omega(\equiv 2 V M)$

$$
M_{G_{0} \rightarrow 2 V M}^{(0) S S C}=\frac{1}{\sqrt{3}} M_{G_{0} \rightarrow 2 P S}^{(0) S S C}
$$

3) $G_{0} \rightarrow \sigma \sigma$

$$
M_{G_{0} \rightarrow \sigma \sigma}^{(0) S S C}=\sqrt{\frac{E_{\sigma} E_{\sigma}}{M_{f_{0}}}} \frac{64 \sqrt{2}}{9} \pi^{7 / 4} \frac{R_{G_{0}}^{7 / 2}\left(2 R_{G_{0}}^{2}-9 R_{B}^{2}\right)}{\left(R_{B}^{2}+2 R_{G_{0}}^{2}\right)^{7 / 2}} K^{1 / 2}
$$

4) $G_{0} \rightarrow \pi^{*}(1300) \pi$

$$
M_{G_{0} \rightarrow \pi^{*} \pi}^{(0) S S C}=\sqrt{\frac{E_{\pi}^{*} E_{\pi}}{M_{f_{0}}}} \frac{32}{3 \sqrt{3}} \pi^{7 / 4} \frac{R_{G_{0}}^{3 / 2} R_{B}^{2}\left(14 R_{G_{0}}^{2}-3 R_{B}^{2}\right)}{\left(R_{B}^{2}+2 R_{G_{0}}^{2}\right)^{7 / 2}} K^{1 / 2}
$$


[1] F.E. Close, Rep. Prog. Phys. 51, 833 (1988).

[2] G. Bali et al., Phys. Lett. B309, 378 (1993); H. Chen, J. Sexton, A. Vaccarino and D. Weingarten, Nucl. Phys. B (Proc. Suppl.) 34, 357 (1994).

[3] M. Teper, hep-ph/9711299.

[4] C. Amsler and F.E. Close, Phys. Lett. B353, 385 (1996); Phys. Rev. D53, 295 (1996);

F.E. Close, Nucl. Phys. B (Proc. Suppl.) 56A, 248 (1997).

[5] D. Weingarten, Nucl. Phys. B (Proc. Suppl.) 53, 232 (1997).

[6] A.V. Anisovich, V.V. Anisovich and A.V. Sarantsev, Phys. Lett. B395, 123 (1997).

[7] S. Narison, Nucl. Phys. B509, 312 (1998).

[8] C. Amsler, Rev. Mod. Phys. 70, 1293 (1998).

See also: D. V. Bugg, A. V. Sarantsev, hep-ex/97120007.

[9] D. V. Bugg et al., Phys. Lett. B353, 378 (1995); W. Dunwoodie, SLAC-PUB-7163, Proc. of Hadron 97 (Upton N. Y. August 1997), p.753; S. J. Lindenbaum and R. S. Longacre, Phys. Lett. B274, 493 (1992).

[10] C. Caso et al., The European Physical Journal C3, 1 (1998); R. M. Barnett et al., Phys. Rev. D54, 1 (1996).

[11] R. Kokoski and N. Isgur, Phys. Rev. D35, 907 (1987).

[12] Godfrey and N. Isgur, Phys. Rev. D32, 189 (1985).

[13] M. Strohmeier-Prešiček, T. Gutsche, R. Vinh Mau and Amand Faessler, Phys. Lett. B438, 21 (1998).

[14] W.Lee and D. Weingarten, hep-lat/9805029. 
[15] U. Thoma, Nucl. Phys. B (Proc. Suppl.) 56A, 216 (1997).

[16] E. S. Ackley and T. Barnes, Phys. Rev. D54, 6811 (1996);

P. Geiger and E. S. Swanson, Phys. Rev. D50, 6855 (1994).

[17] A. Le Yaouanc et al., Hadron transitions in the quark model, Gordon and Breach, Amsterdam (1988).

[18] M. Maruyama, S. Furui and Amand Faessler, Nucl. Phys. A472, 643 (1987)

[19] M. Nagels et al., Phys. Rev. D12, 744 (1975).

[20] U. Thoma, Proc. of Hadron 97 (Upton N. Y. August 1997), p. 322.

[21] N. Isgur and J. Paton, Phys. Rev. D31, 2910 (1985).

[22] H. G. Dosch and D. Gromes, Phys. Rev. D33, 1278 (1986).

[23] T. Gutsche et al., to be published.

[24] C. Amsler et al., Phys. Lett. B294, 451 (1992).

[25] R. L. Jaffe and K. Johnson, Phys. Lett. 60B, 201 (1976); Phys. Rev. Lett. 34, 1645 (1976).

[26] A. Le Yaouanc et al., Z. Phys. C28, 309 (1985); F. Iddir et al., Phys. Lett. B205, 564 (1988).

[27] M. Moshinsky, The harmonic oscillator in modern physics: from atoms to quarks, Gordon and Breach, New York (1969); T. A. Brody and M. Moshinsky, Tables of transformation brackets, Gordon and Breach, New York (1967).

[28] D. V. Bugg, A. V. Sarantsev, B. S. Zou, Nucl. Phys. B471, 59 (1996); A. Abele et al., Nucl. Phys. A609, 562 (1996).

[29] J. Sexton, A. Vaccarino and D. Weingarten, Phys. Rev. Lett. 75, 4563 (1995); Nucl. Phys. Proc. Suppl. 47, 128 (1996).

[30] V. V. Anisovich et al., Phys. Lett. B364, 195 (1995). 
[31] H. Jin and X. Zhang, hep-ph 19805412. 
FIG. 1. Leading order decay mechanism of the $G_{0} \rightarrow G_{0} G_{0}$ transition. The dot indicates the three-gluon coupling.

FIG. 2. Quark line diagram for the decay of the quarkonia component $Q \bar{Q}$ into the final state mesons BC occurring as a leading order decay mechanism in strong coupling QCD. The dot indicates the ${ }^{3} P_{0}$ vertex with strength $\lambda$.

FIG. 3. Transition $G_{0} \rightarrow B C$ occurring as a next-to-leading order decay mechanism in strong coupling QCD. The dots indicate the quark-gluon coupling with strength $\gamma$.

FIG. 4. Dependence of the branching ratios $B R\left(f_{0}(1500) \rightarrow B C\right)$ on the mixing angle $\alpha$, defined by the $Q \bar{Q}$ configuration $\left|f_{0}(1500)>=\cos \alpha\right| n \bar{n}>-\sin \alpha \mid s \bar{s}>$, for the decay mechanism $Q \bar{Q} \rightarrow B C$ of Fig. 2. For the meson size parameters we take the values given in the main text. The arrows indicate the predictions for the mixing schemes of Refs. [4.5] in lowest order of the decay mechanism.

FIG. 5. Dependence of the total width of the $f_{0}(1500)$ resonance on the mixing angle $\alpha$ defined by the $Q \bar{Q}$ configuration $\left|f_{0}(1500)>=\cos \alpha\right| n \bar{n}>-\sin \alpha \mid s \bar{s}>$. The shadowed band corresponds to the experimental total width of $\Gamma=111 \pm 12 \mathrm{MeV}$ [8].

FIG. 6. Dependence of the branching ratios $B R\left(G_{0} \rightarrow B C\right)$ on the glueball mass for the decay mechanism $G_{0} \rightarrow B C$ of Fig. 3. Exact flavor $S U(3)$ symmetry for the quark-gluon vertex is assumed. For the size parameters we use the values given in the main text. For mass averaging we take a glueball width of $\Gamma_{G_{0}}=120 \mathrm{MeV}$. 
TABLE I. Flavor matrix elements for the two-body decay $Q \bar{Q} \rightarrow B C$ of the mixed $f_{0}(M)$ states as defined in Eq. (11). The quarkonia component of the $f_{0}$ states is defined as $\left|Q \bar{Q}>=a_{n \bar{n}}\right| n \bar{n}>+a_{s \bar{s}} \mid s \bar{s}>$. The quantities $\alpha_{P S}$ and $\beta_{P S}$ are related to the pseudoscalar mixing angle as defined by Eq. (13). For the vector mesons we use ideal mixing.

\begin{tabular}{ll}
\hline \hline $\mathrm{BC}$ & $M_{Q \bar{Q} \rightarrow B C}^{F}$ \\
\hline$\pi \pi, \rho \rho$ & $\sqrt{3} a_{n \bar{n}}$ \\
$\sigma \sigma, \omega \omega$ & $a_{n \bar{n}}$ \\
$\pi^{*}(1300) \pi, a_{1} \pi$ & $\sqrt{6} a_{n \bar{n}}$ \\
$K \bar{K}$ & $a_{n \bar{n}}+\sqrt{2} a_{s \bar{s}}$ \\
$\eta \eta$ & $\alpha_{P S}^{2} a_{n \bar{n}}+\sqrt{2} \beta_{P S}^{2} a_{s \bar{s}}$ \\
$\eta \eta^{\prime}$ & $2 \alpha_{P S} \beta_{P S}\left(a_{n \bar{n}} / \sqrt{2}-a_{s \bar{s}}\right)$ \\
\hline \hline
\end{tabular}


TABLE II. Flavor matrix elements of Eq. (21) for the two-body decay $G_{0} \rightarrow B C$ of the mixed $f_{0}(M)$ states with glueball component $a_{G_{0}} \mid G_{0}>$. The quark-gluon coupling is assumed to be flavor independent.

\begin{tabular}{lc}
\hline \hline $\mathrm{BC}$ & $M_{G_{0} \rightarrow B C}^{F} / a_{G_{0}}$ \\
\hline$\pi \pi, \rho \rho$ & $\sqrt{6}$ \\
$\sigma \sigma, \omega \omega$ & $\sqrt{2}$ \\
$\pi^{*}(1300) \pi, a_{1} \pi$ & $\sqrt{12}$ \\
$K \bar{K}$ & $2 \sqrt{2}$ \\
$\eta \eta$ & $\sqrt{2}$ \\
$\eta \eta^{\prime}$ & 0 \\
\hline \hline
\end{tabular}


TABLE III. Squared decay amplitudes and interference terms as defined by Eq. (5) for the transition $f_{0}(1500) \rightarrow B C$. Mass averaging as in Eq. (6) is already included. For the interference term we have $l_{B C}=0$ as restricted by the dynamical selection rule of the $G_{0} \rightarrow B C$ transition. All values are given in $\mathrm{MeV}$.

\begin{tabular}{llll}
\hline \hline BC & $M_{Q \bar{Q} \rightarrow B C}^{2}$ & $M_{G_{0} \rightarrow B C}^{2}$ & $M_{G_{0} \rightarrow B C}^{(0)} M_{Q \bar{Q} \rightarrow B C}^{(0)}$ \\
\hline$\pi \pi$ & $52.2 a_{n \bar{n}}^{2}$ & $224.4 a_{G_{0}}^{2}$ & $-103.1 a_{n \bar{n}} a_{G_{0}}$ \\
$\eta \eta$ & $20.9\left(0.63 a_{n \bar{n}}+0.523 a_{s \bar{s}}\right)^{2}$ & $865.8 b_{G_{0}}^{2}$ & $-131.1 a_{G_{0}}\left(0.63 a_{n \bar{n}}+0.523 a_{s \bar{s}}\right)$ \\
$\eta \eta^{\prime}$ & $10.8\left(a_{n \bar{n}} / \sqrt{2}-a_{s \bar{s}}\right)^{2}$ & 0 & 0 \\
$K \bar{K}$ & $10.7\left(a_{n \bar{n}}+\sqrt{2} a_{s \bar{s}}\right)^{2}$ & $828.0 a_{G_{0}}^{2}$ & $-86.8 a_{G_{0}}\left(a_{n \bar{n}}+\sqrt{2} a_{s \bar{s}}\right)$ \\
$\rho \rho$ & $50.4 a_{n \bar{n}}^{2}$ & $15.0 a_{G_{0}}^{2}$ & $10.2 a_{n \bar{n}} a_{G_{0}}$ \\
$\omega \omega$ & $5.3 a_{n \bar{n}}^{2}$ & $2.2 a_{G_{0}}^{2}$ & $2.0 a_{n \bar{n}} a_{G_{0}}$ \\
$a_{1} \pi$ & $94.2 a_{n \bar{n}}^{2}$ & 0 & 0 \\
$\sigma \sigma$ & $16.1 a_{n \bar{n}}^{2}$ & $264.6 a_{G_{0}}^{2}$ & $-63.1 a_{n \bar{n}} a_{G_{0}}$ \\
$\pi^{*}(1300) \pi$ & $7.2 a_{n \bar{n}}^{2}$ & $440.4 a_{G_{0}}^{2}$ & $-55.2 a_{n \bar{n}} a_{G_{0}}$ \\
\hline \hline
\end{tabular}


TABLE IV. Predictions of mixing scheme A 画 for the partial decay widths $\Gamma(B C)$ of the physical $f_{0}$ states, $\mathrm{B} \mathrm{C}$ are the final state mesons. The $4 \pi$ decay channel refers to the contributions of the $\sigma \sigma$ and $\pi^{*} \pi$ intermediate states using $B R\left(\pi^{*} \rightarrow \sigma \pi\right)=0.1-1$. All values are given in $\mathrm{MeV}$. The predicted ratios $B R\left(f_{0}(1370) \rightarrow K \bar{K}\right) / B R\left(f_{0}(1370) \rightarrow \eta \eta\right)=2.6$ and $B R\left(f_{0}(1710) \rightarrow \pi \pi\right) / B R\left(f_{0}(1710) \rightarrow K \bar{K}\right)=0.93$ have to be compared to the experimental values of $\approx 2.5[8]$ and $0.39 \pm 0.14$ [10].

\begin{tabular}{|c|c|c|c|c|c|c|c|c|c|c|c|}
\hline & $\Gamma_{t o t}$ & $\pi \pi$ & $K \bar{K}$ & $\eta \eta$ & $\eta \eta^{\prime}$ & $\rho \rho$ & $\omega \omega$ & $a_{1} \pi$ & $\pi^{*} \pi$ & $\sigma \sigma$ & $4 \pi$ \\
\hline \multicolumn{12}{|c|}{$f_{0}(1500)$} \\
\hline Model & 136 & 29.3 & 3.9 & 5.2 & 19 & 19 & 2 & 36.8 & 9.9 & 12.5 & $13.5-22.4$ \\
\hline Exp. [8] & $111 \pm 12$ & $32 \pm 8.4$ & $3.9 \pm 0.3$ & $5.2 \pm 1.5$ & $1.4 \pm 0.4$ & & & & & & $68 \pm 10.7$ \\
\hline \multicolumn{12}{|c|}{$f_{0}(1370)$} \\
\hline Model & 410 & 65.4 & 29.1 & 10.8 & 3 & 112.1 & 22.8 & 138.6 & 12.7 & 16 & $17.3-28.7$ \\
\hline Exp. 8 & $351 \pm 41$ & & $K \bar{K} / \eta \eta$ & & & & & & & & \\
\hline \multicolumn{12}{|c|}{$\Lambda \Lambda / T \eta \approx 2 . J$} \\
\hline Model & 179 & 23.4 & 25.1 & 22.6 & 20.3 & 21.3 & 4.3 & 28.2 & 20.9 & 12.7 & $14.8-33.6$ \\
\hline Exp. 10 & $133 \pm 14$ & \multicolumn{10}{|c|}{$\pi \pi / K \bar{K}=0.39 \pm 0.14$} \\
\hline
\end{tabular}


TABLE V. Predictions of mixing scheme B [5] for the partial decay widths $\Gamma(B C)$ of the physical $f_{0}$ states. The $4 \pi$ decay channel refers to the contributions of the $\sigma \sigma$ and $\pi^{*} \pi$ intermediate states using $B R\left(\pi^{*} \rightarrow \sigma \pi\right)=0.1-1$. All values are given in $\mathrm{MeV}$. The predicted ratios $B R\left(f_{0}(1370) \rightarrow K \bar{K}\right) / B R\left(f_{0}(1370) \rightarrow \eta \eta\right)=1.45$ and $B R\left(f_{0}(1710) \rightarrow \pi \pi\right) / B R\left(f_{0}(1710) \rightarrow K \bar{K}\right)=0.60$ have to be compared to the experimental values of $\approx 2.5[8]$ and $0.39 \pm 0.14$ [10].

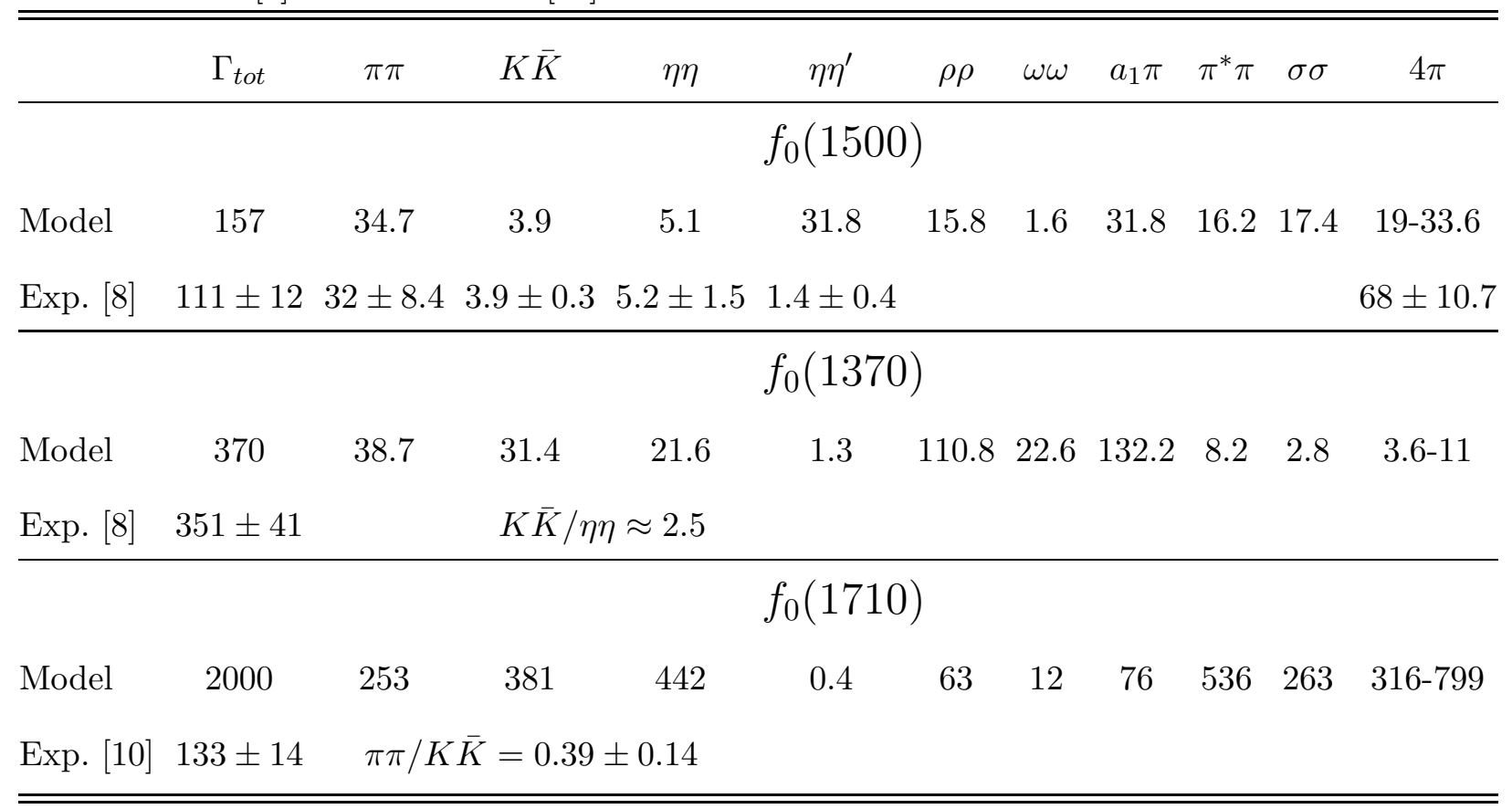


TABLE VI. Predictions for the partial decay widths $\Gamma(B C)$ of the physical $f_{0}$ states for the extracted mixing scheme. The $4 \pi$ decay channel refers to the contributions of the $\sigma \sigma$ and $\pi^{*} \pi$ intermediate states using $B R\left(\pi^{*} \rightarrow \sigma \pi\right)=0.1-1$. All values are given in $\mathrm{MeV}$. The predicted ratios $B R\left(f_{0}(1370) \rightarrow K \bar{K}\right) / B R\left(f_{0}(1370) \rightarrow \eta \eta\right)=2.7$ and $B R\left(f_{0}(1710) \rightarrow \pi \pi\right) / B R\left(f_{0}(1710) \rightarrow K \bar{K}\right)=0.57$ have to be compared to the experimental values of $\approx 2.5[8]$ and $0.39 \pm 0.14[10]$.

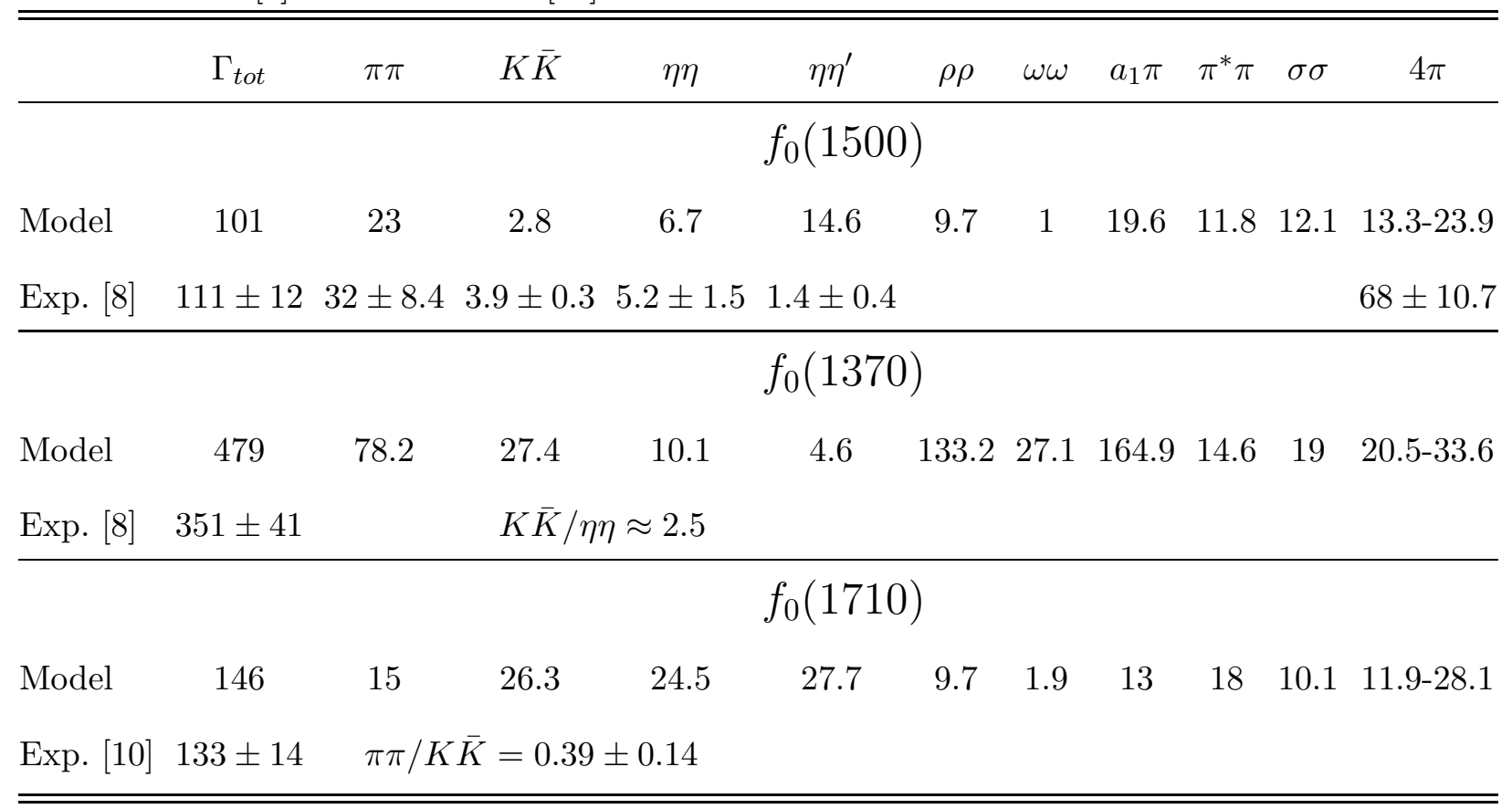


Fig. 1

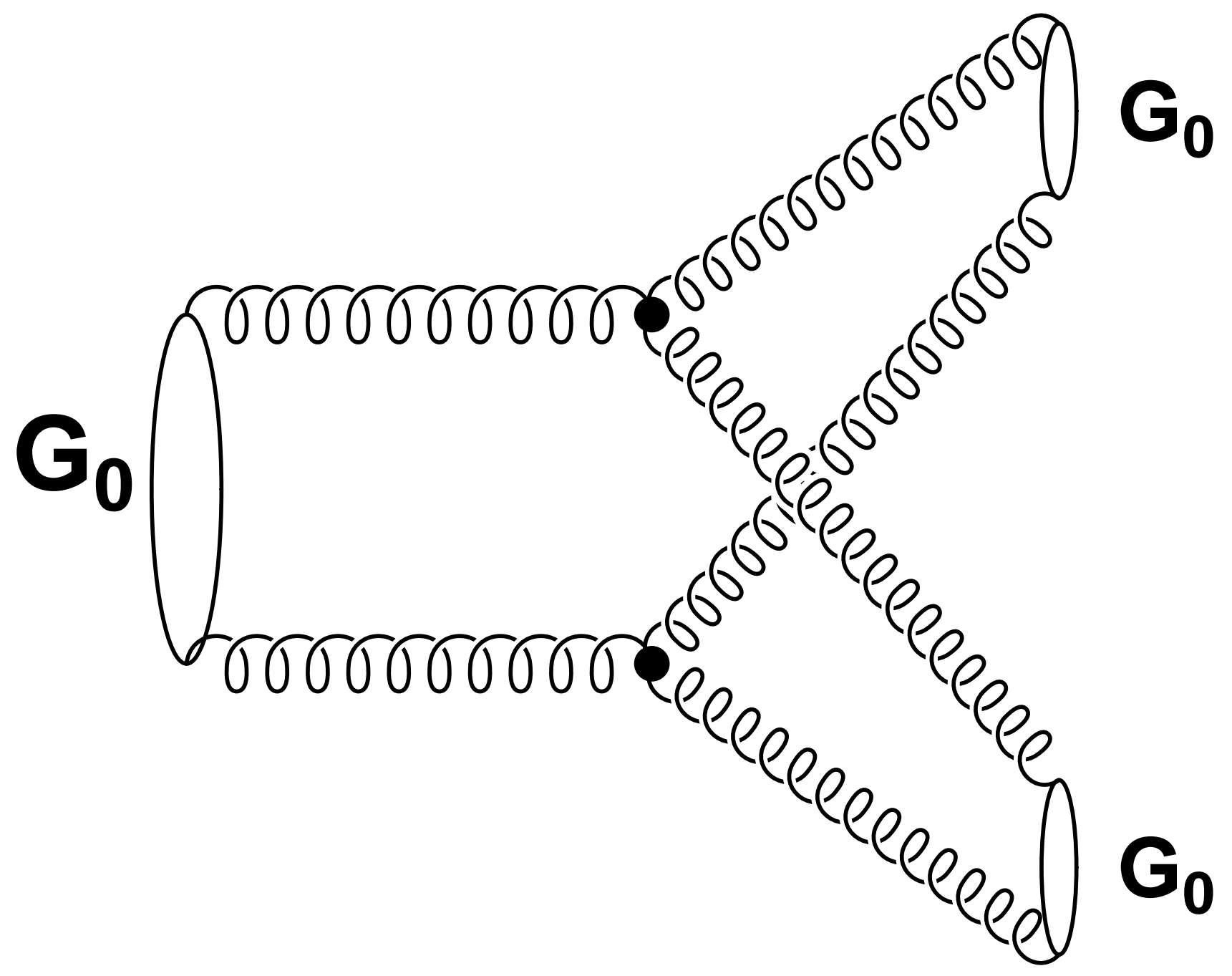


Fig. 2

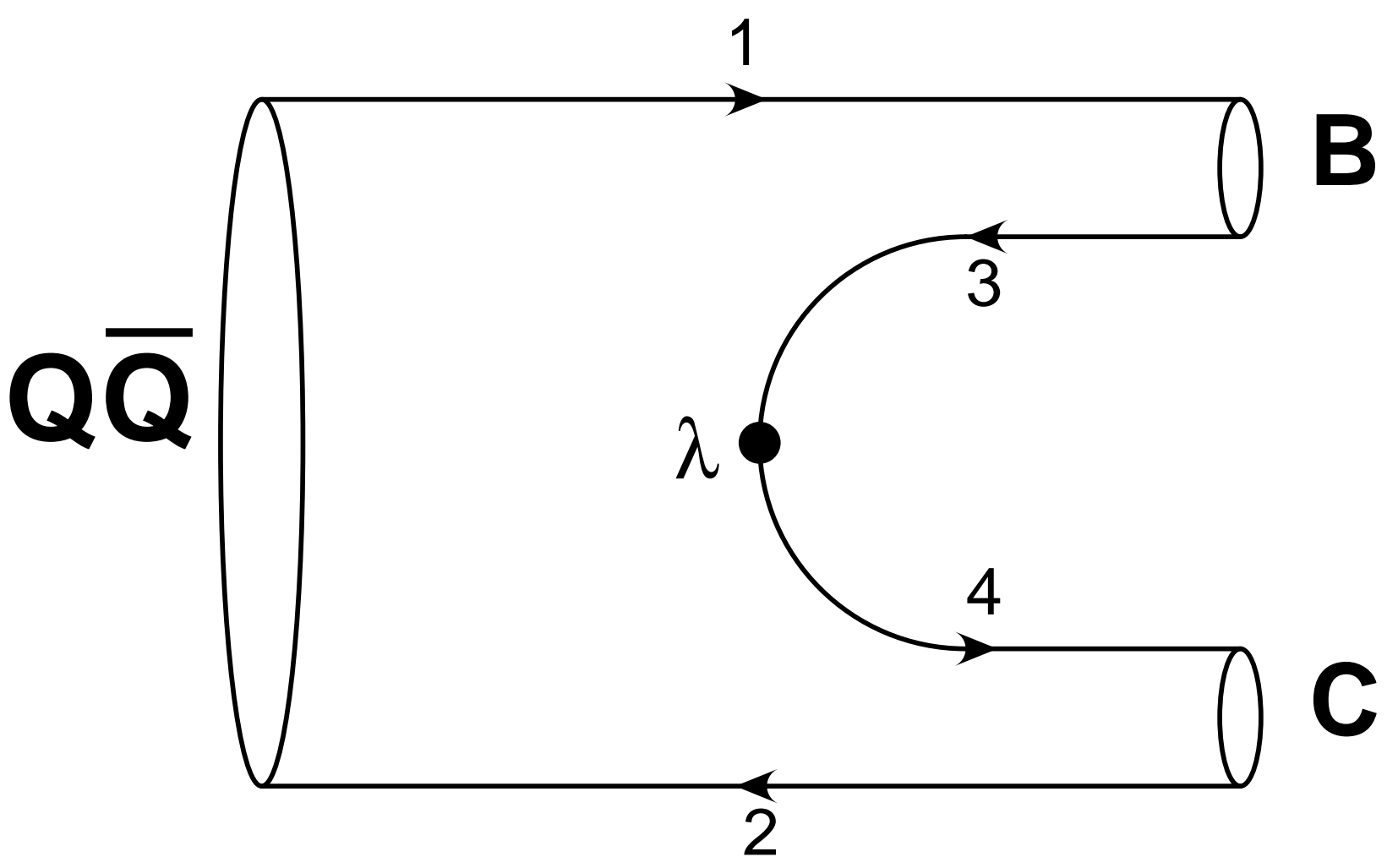


Fig. 3

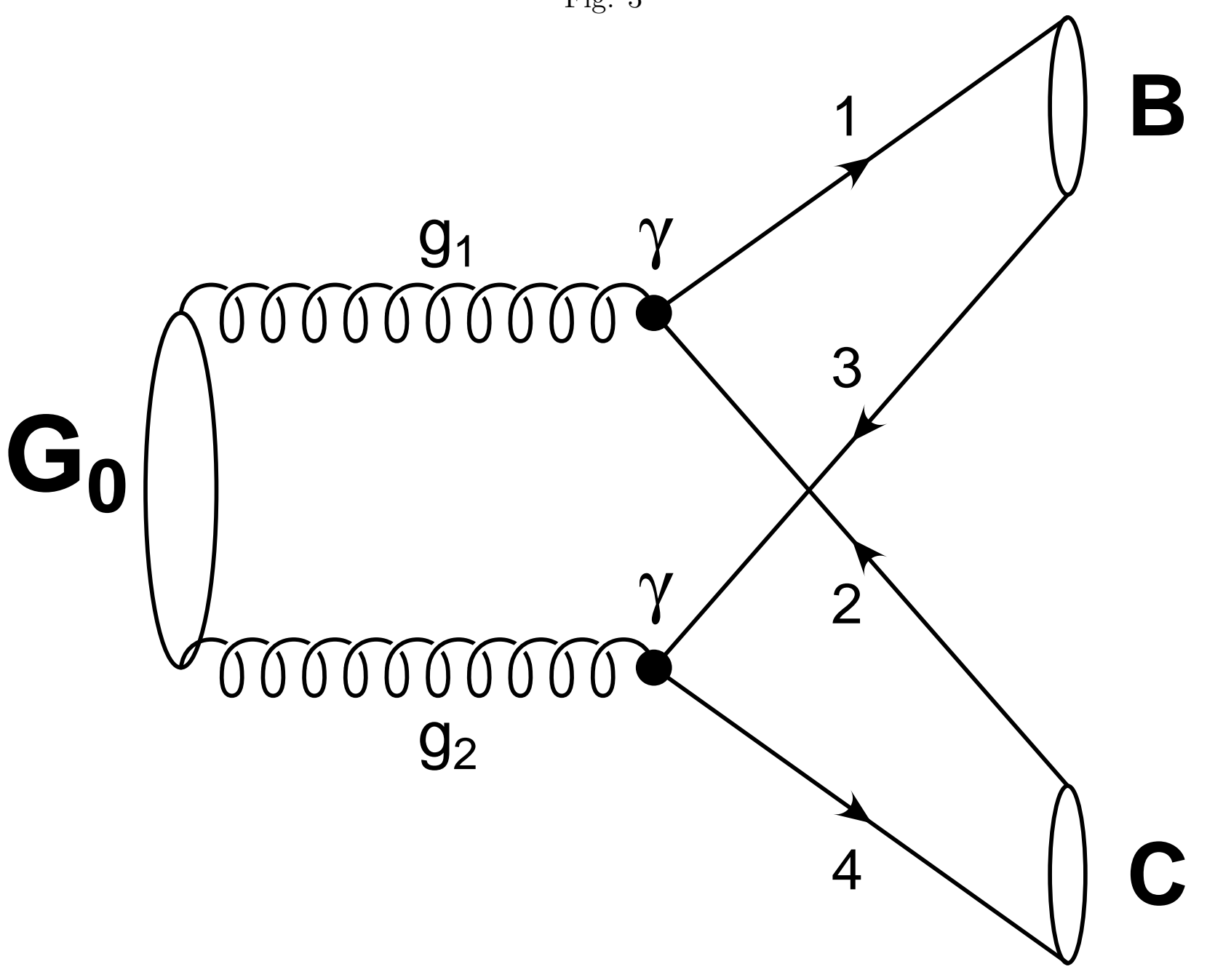


Fig. 4

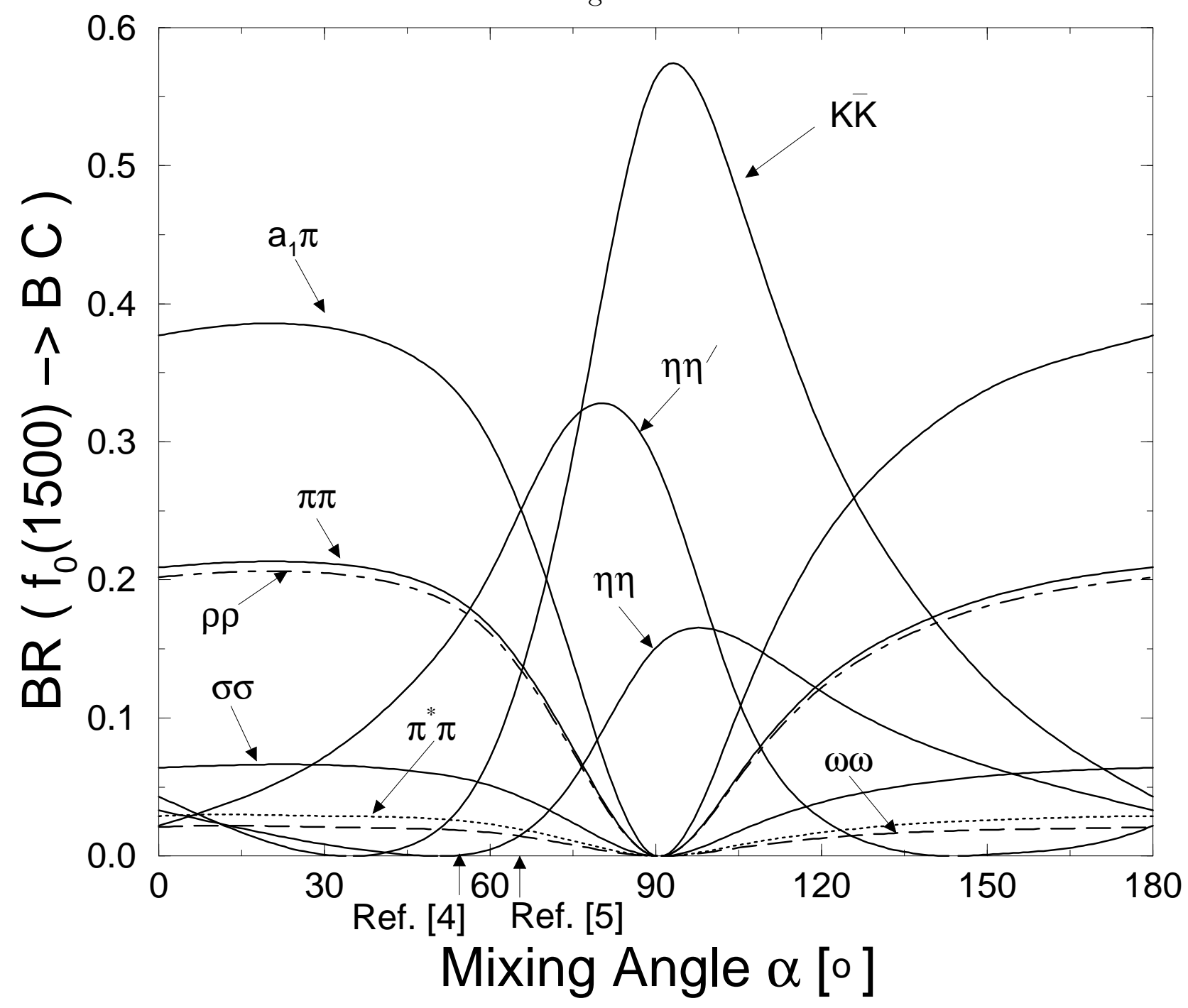


Fig. 5

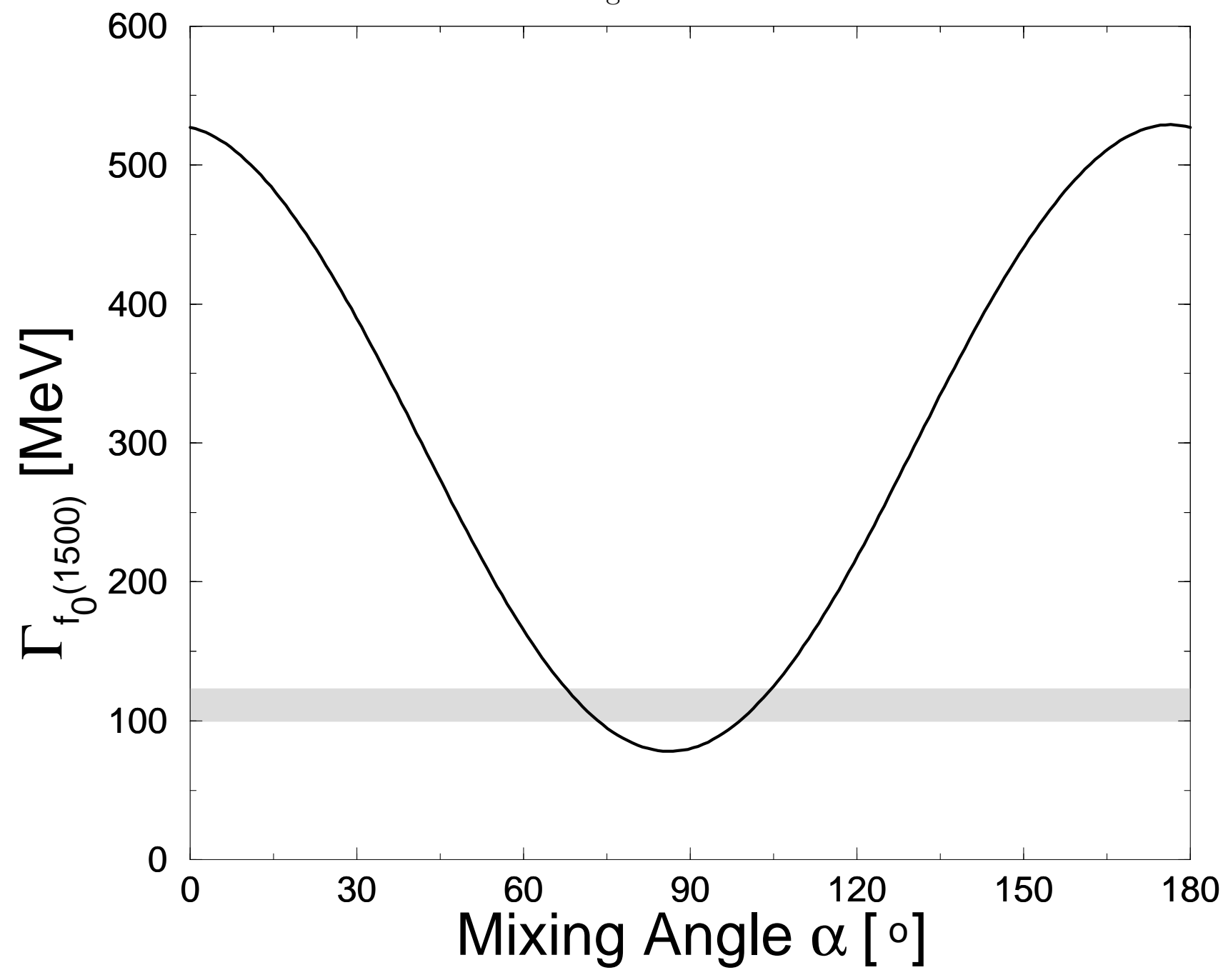


Fig. 6

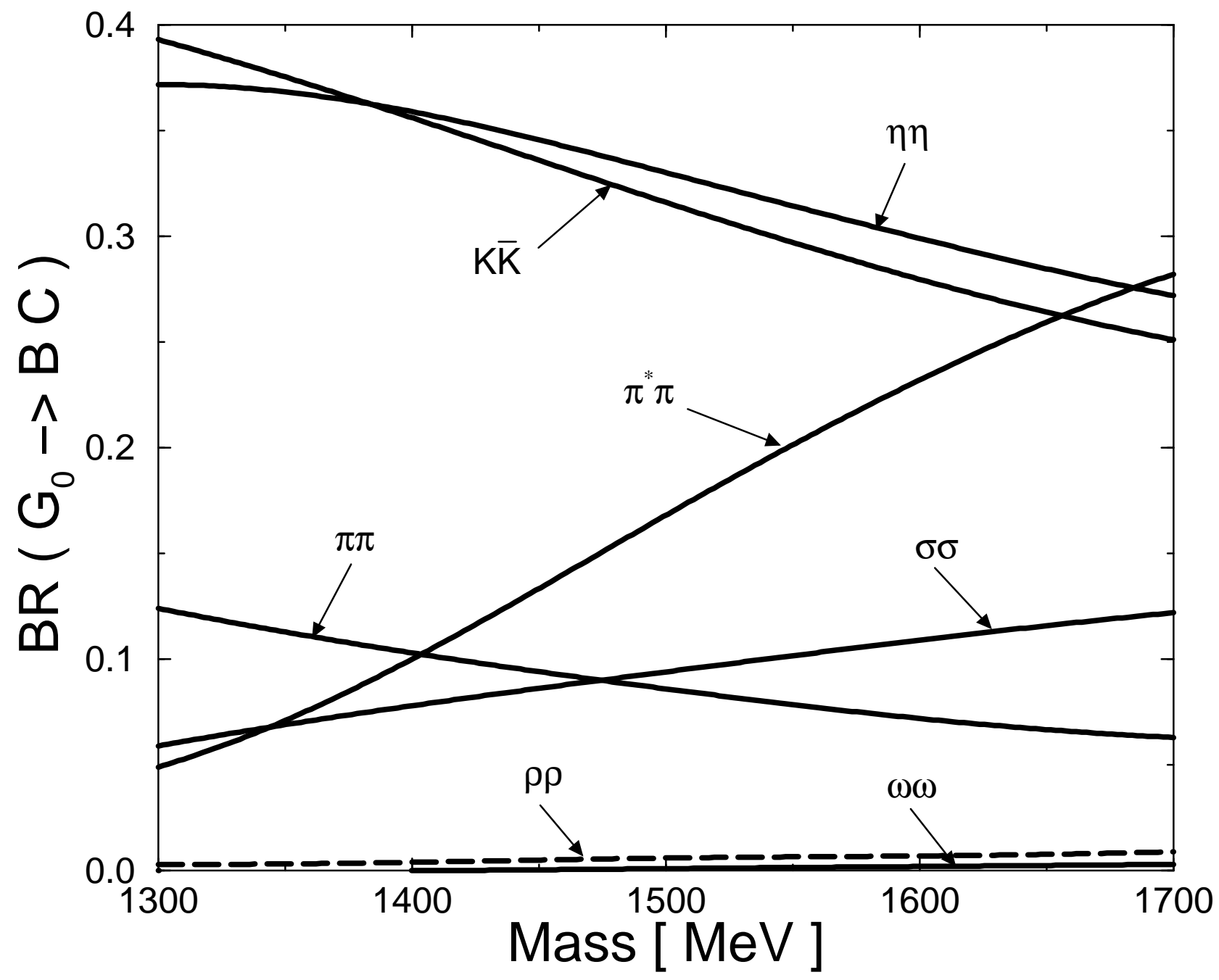

